Luigi BISIO*

\title{
I Coleotteri Carabidi della Valpelline (Valle d'Aosta) (Coleoptera Carabidae)
}

\begin{abstract}
Riassunto: L'autore espone una sintesi della storia delle ricerche entomologiche in Valpelline (Valle d'Aosta, Alpi Pennine) con particolare attenzione ai precursori. Le prime indagini in questo territorio furono svolte da alcuni coleotterologi svizzeri (Bischoff-Ehinger, Stierlin, Born e Gaud) tra la fine dell'Ottocento e l'inizio del secolo scorso. Poi, durante la prima metà del Novecento, le conoscenze sulla coleotterofauna che occupa questa valle furono notevolmente incrementate dall'Abate Henry (Abbé Henry), parroco del paese di Valpelline. Formidabile scalatore e infaticabile escursionista, egli raccolse Coleotteri nei luoghi più inaccessibili della valle contribuendo così a formare un primo gruppo consistente di dati corologici relativi a quest'area. Dopo aver brevemente illustrato i principali caratteri geologici, climatici e vegetazionali della valle, l'autore presenta un catalogo topografico delle 141 specie di Carabidi (Cicindelinae incluse) segnalate in questo territorio sino a oggi, con note riguardanti l'ecologia e la corologia di quelle più interessanti. Infine, sono descritte le principali carabidocenosi osservate.
\end{abstract}

\begin{abstract}
Carabid beetles of Valpelline valley (Valle d'Aosta) (Coleoptera Carabidae).
An outline of the history of the entomological researches in Valpelline valley (Valle d'Aosta, Pennine Alps, Italy), with particular attention to the precursors, is presented. The initial investigations in this territory were carried out by Swiss coleopterists (Bischoff-Ehinger, Stierlin, Born and Gaud) between the end of the nineteenth century and the beginning of the last century. Subsequently, during the first half of the twentieth century, the knowledge of the beetle fauna living in this valley was significantly increased by Abbot Henry (Abbé Henry), the parish priest of the village of Valpelline. Formidable climber and indefatigable hiker, he collected beetles in the most inaccessible sites of the valley, thus contributing to form a first substantial group of chorological data related to this area. After a short illustration of the main geological as well as climatic and vegetational features of the Valpelline valley, a topographic catalogue of the 141 Carabid species (Cicindelinae included) recorded so far from this territory is also given, with notes regarding the ecology and the distribution of the most interesting ones. Furthermore, the main observed carabid assemblages are described.
\end{abstract}

Key words: Coleoptera Carabidae, Western Alps, Valpelline, Valle d'Aosta.

\section{INTRODUZIONE}

Con questa nota dedicata alla Valpelline, lo scrivente continua la serie dei contributi che diversi autori hanno recentemente dedicato ai Carabidi (Cicindelinae incluse) di alcune valli o aree protette valdostane (Allegro \& Bisio, 2007; Allegro \& Chiarabaglio, 2008; Allegro et al., 2011; Bisio, 2006, 2013b, Bisio et al., 2012, in stampa; Bisio \& Giuntelli, 2014). La valle è stata esplorata da generazioni di entomologi e il suo territorio è stato oggetto di numerose segnalazioni faunistiche riguardanti soprattutto i Coleotteri. Già nel corso della seconda metà dell'Ottocento e della prima metà del secolo scorso, elenchi più o meno lunghi di Coleotteri raccolti nella valle in oggetto furono pubblicati da diversi autori che vi avevano effettuato ricerche nel corso di escursioni spesso lunghe e faticose. Molti di questi lavori, tuttavia, in Italia sono stati per lungo tempo pressoché scono- sciuti, perché pubblicati su riviste straniere o su riviste che all'epoca avevano una limitata diffusione territoriale. Non è un caso che né il catalogo topografico di Magistretti (1965), né la più recente CKmap (Casale et al., 2006) abbiano citato per la Valpelline i dati corologici forniti da questi autori. Oggi, grazie a Internet e ai suoi motori di ricerca, è possibile consultare molto più facilmente queste pubblicazioni. Ciò ha offerto l'occasione allo scrivente di rivisitare i lavori un po' dimenticati di questi precursori e di ripercorrere idealmente gli itinerari delle loro escursioni.

I primi contributi alla conoscenza dei Coleotteri che popolano il territorio in oggetto furono merito di entomologi svizzeri. Infatti, in un'epoca nella quale le valli alpine si attraversavano molto spesso a piedi, la Valpelline era un frequente luogo di transito poiché, oltre al Passo del Gran San Bernardo (nella vicina valle omonima), anche la Fenêtre de Durand (Fig. 1) (citato

*Luigi Bisio, Via Galilei 4, 10082 Cuorgnè (TO), Italia. E-mail: luigibisio@virgilio.it 
spesso semplicemente come «Col Fenêtre» dai diversi autori), ampio colle situato alla testata del Vallone di Ollomont, costituiva un comodo valico per i viandanti d'oltralpe che intendevano raggiungere Aosta o che volevano rientrare in patria. Al colle citato, durante un viaggio effettuato a scopo coleotterologico nel Biellese e in Valle d'Aosta, salirono Andreas Bischoff-Ehinger e Gustav Stierlin (rispettivamente di Basilea e di Sciaffusa). I due entomologi, in un accurato resoconto sulle specie raccolte durante la loro «Reise» (Bischoff-Ehinger \& Stierlin, 1870), segnalarono diversi Carabidi raccolti nella Conca di By e, più in generale, in Valpelline. Poi, nell'estate del 1899, fu di passaggio in valle Paul Born carabidologo ("der Käfer-Doktor") di Herzogenbuchsee (Cantone di Berna), vero precursore delle ricerche entomologiche nelle valli alpine che egli attraversava con lunghe escursioni a piedi (oggi si parlerebbe di trekking). Come descrisse accuratamente in due delle numerose note dedicate alle sue «Excursion» (Born, 1900a, 1900b), nell'estate del 1899 egli giunse a Nus dopo un viaggio di più giorni (proveniva infatti dalla Savoia). Da qui, risalì la Valle di Saint-Barthélemy e, attraverso il colle omonimo, discese (lungo la Comba Verdona) prima a Oyace e poi a Valpelline dove per-

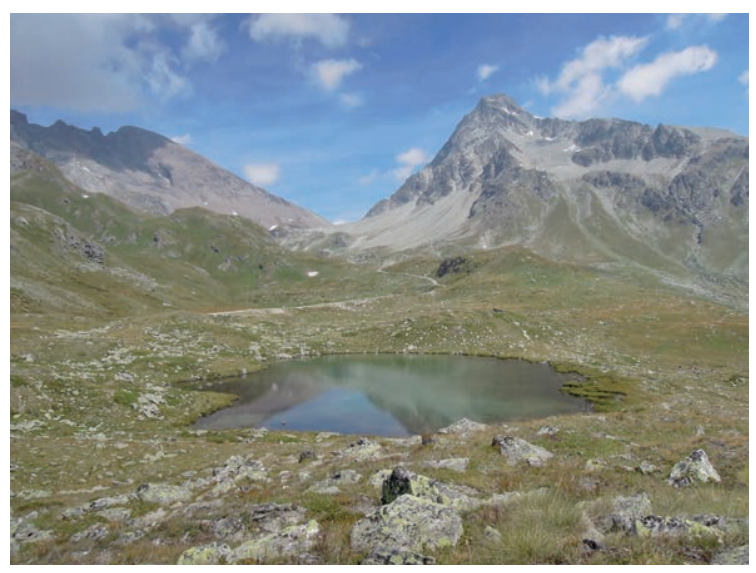

Fig. 1. Il Col Fenêtre de Durand visto dai Lacs de Thoules (14.VIII.2012). Gli ambienti intorno ai laghi sono l'habitat di una carabidocenosi orofila ricca di specie (Cicindela campestris, C. gallica, Carabus concolor, Nebria laticollis, Oreonebria castanea, Bembidion bipunctatum, B. geniculatum, B. pyrenaeum, B. glaciale, B. magellense, B. incognitum, Pterostichus multipunctatus, Amara erratica, $A$. praetermissa, A. quenseli, Harpalus fuliginosus, Calathus melanocephalus, C. erratus e Cymindis vaporariorum). nottò. Il giorno seguente, percorrendo il Vallone di Ollomont, rientrò in Svizzera attraverso il Col Fenêtre. Il resoconto delle due giornate è corredato dagli elenchi piuttosto corposi dei Coleotteri raccolti. Qualche anno dopo, fu Alphonse Gaud (di Montreux) a relazionare (Gaud, 1902) sui Coleotteri rinvenuti nel corso un'escursione («course») effettuata ad Aosta tra il luglio e l'agosto del 1902, valicando lo stesso colle e attraversando la Valpelline, insieme ad alcuni membri dell'associazione naturalistica "La Murithienne du Valais".

Tutte le segnalazioni di questi autori furono poi riprese da Pavesi (1904) nella sua sintesi monografica sulla fauna della Valle d'Aosta.

Nella prima metà del Novecento, le conoscenze sulla coleotterofauna del territorio in oggetto furono notevolmente incrementate grazie alle ricerche dell'Abate Joseph Marie Henry (Abbé Henry), attivo in valle dal 1903, anno nel quale fu nominato parroco del paese di Valpelline. Alpinista formidabile, autore di numerose ascensioni sulle più importanti vette valdostane e su gran parte delle cime della Valpelline, egli coniugava questa passione con quella del naturalista. Fu soprattutto botanico (con particolare predilezione per la lichenologia), ma durante le sue numerose escursioni nei valloni più impervi della Valpelline non trascurava di controllare anno dopo anno lo stato dei ghiacciai (stato sul quale poi relazionava con regolarità) e di raccogliere insetti (soprattutto Coleotteri, tra i quali molti Carabidi). Il materiale entomologico ottenuto - cartellinato in modo molto dettagliato riportando scrupolosamente la frazione, il vallone («Combe») o il monte nei pressi dei quali il singolo esemplare era stato raccolto - lo inviava poi a specialisti svizzeri dell'epoca per la determinazione; ciò gli permise la pubblicazione sul "Bulletin de la Société de la Flore Valdôtaine" di quattro cataloghi topografici delle specie rinvenute (Henry, 1915, 1916, 1935, 1938). Sebbene questi lavori non siano del tutto scevri da inesattezze (alcune molto evidenti: come, ad esempio, la segnalazione di «Pterostichus impressus Fairm.», specie che oggi sappiamo diffusa dalle Alpi Liguri alle Cozie; la sua peculiare morfologia rende persino difficile intuire quale specie effettivamente presente in valle possa essere stata confusa con questa entità e correggere di conseguenza la segnalazione), la maggior parte delle numerose segnalazioni è molto verosimile, perché confermata da recenti reperti ottenuti in Valpelline o in altre valli valdostane limitrofe. È pur vero che, per quanto riguarda i Carabidi, il quadro faunistico che emerge dalla lettura dei suoi lavori è par- 
zialmente incompleto, soprattutto per quanto attiene alla fauna ripicola: molto frammentari, infatti, sono i dati sui Bembidiini e non è mai citata Nebria laticollis, entità la cui presenza è stata accertata in diverse stazioni (v. Catalogo topografico). D'altronde, da un appassionato botanico che s'interessava in modo relativamente marginale anche di Coleotteri (molti dei quali trovati probabilmente per caso durante le sue escursioni) non si potevano pretendere ricerche entomologiche specializzate. Rimane comunque il fatto che i suoi elenchi costituiscono un patrimonio di dati che, quantomeno per i Carabidi, rappresenta il più importante contributo alla conoscenza della fauna presente in Valpelline.

Quasi contemporaneamente ai lavori di Henry, un piccolo contributo fu fornito anche da Schatzmayr (1930) che segnalò la presenza in valle di due specie di Pterostichus.

Nella seconda metà del Novecento, dopo alcuni dati riportati da Magistretti (1965) nel suo già citato catalogo, il contributo più significativo è stato quello di Ravizza (1972) che, nell'ambito di una serie di campionamenti effettuati in Valle d'Aosta allo scopo di rilevare la fauna dei Bembidiini presente in questo territorio, ha elencato le diverse specie rinvenute in quattro stazioni della Valpelline lungo il greto del Torrenti Buthier e Ollomont. Il nucleo di Bembidiini da lui segnalati è, di fatto, un'opportuna integrazione ai dati già numerosi forniti dai lavori di Henry. Successivamente, nuovi dati sono stati forniti dai lavori di Bisio (1999, 2002, 2005, 2008, 2009a, 2009b), Casale et al. (1982, 2006), Focarile (1975), Focarile \& Casale (1978), Sciaky (1987).

Questa nota intende riassumere le conoscenze a tutt'oggi disponibili sulla carabidofauna che popola la Valpelline.

\section{AREA DI STUDIO}

La Valpelline (Fig. 2) si sviluppa lungo il versante sinistro della Valle del Gran San Bernardo (sinistra orografica della Valle d'Aosta) della quale è, di fatto, la più estesa valle laterale. Il suo territorio racchiude l'alto corso del Torrente Buthier sino all'abitato di Roisan nei pressi del quale il torrente stesso riceve le acque dell'Artavanaz, il corso d'acqua che è alimentato dal bacino idrografico del Gran San Bernardo. ${ }^{1}$ La valle, che ha un asse Nord-Est/Sud-Ovest, è addossata al tratto di cresta assiale alpina delimitato a Nord-Est dalla Dent d'Hérens e a Sud-Ovest dal Mont Vélan. Tale tratto (Cresta Dent d'Hérens-Tête de Valpelline-Tête Blanche-Mont Braoulé-Becca d'Oren-Becca Blanchen-Becca RayetteMont Gelé-Mont Avril-Grande Tête de By-Mont Vélan) costituisce l'ossatura della testata e del versante destro della valle e la separa dal territorio svizzero. Lungo la sinistra orografica si snoda invece la dorsale (cresta Dent d'Hérens-Grandes Murailles-Punta Fontanella-Dôme de Tzan-Mont Redessau-Becca di Luseney-Becca d'Arbière-Punta Montagnaya-Becca del Merlo-Monte Pisonet-Monte Faroma-Tsaat à l'Etsena-Mont Mary-Becca di Viou) che divide il territorio in oggetto dalla Valtournenche e dalla Valle di Saint-Barthélemy. Infine, un contrafforte meridionale del Mont Vélan separa l'area in oggetto dalla Valle del Gran San Bernardo. Il torrente

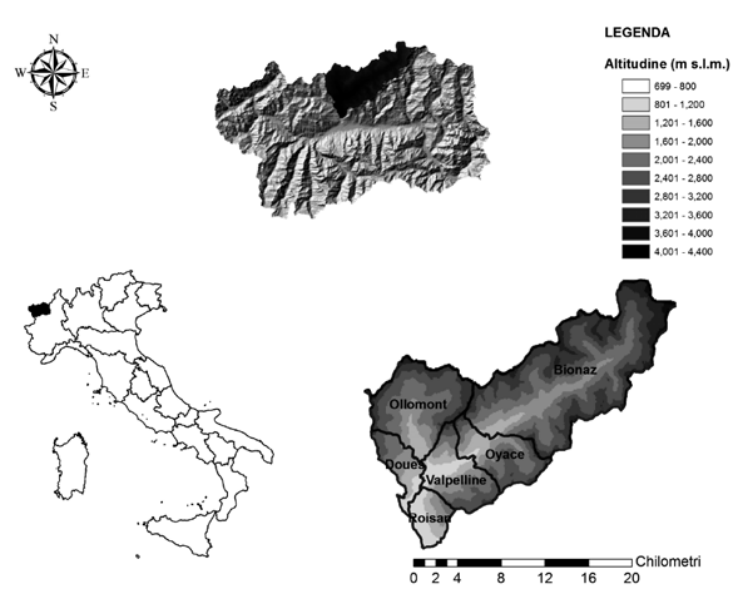

Fig. 2. La Valpelline, territorio oggetto della ricerca (tavola redatta da Matteo Negro).

\footnotetext{
1 Il nome "Buthier" ("Bauticus" per i Romani, da cui il nome "Baltea" attribuito alla Dora) identifica da tempo immemorabile il torrente che, defluendo dalla bassa Valle del Gran San Bernardo, confluisce nella Dora Baltea poco a valle di Aosta. Come già evidenziava Baretti (1893), è un fatto piuttosto anomalo che nell'accezione comune tale nome, a monte della confluenza tra la Valle del Gran San Bernardo (che di fatto è considerata valle principale) e la Valpelline (valle secondaria), sia attribuito al torrente che scorre nella seconda. Denominandolo in tal modo, questo torrente è elevato al rango di asta principale, mentre il corso d'acqua (attualmente denominato Artavanaz) che solca la parte medio alta della Valle del Gran San Bernardo è ridotto a quello di semplice tributario.
} 
principale riceve le acque da diversi valloni tributari; di questi il Vallone di Ollomont è il più importante per estensione. Il territorio vallivo è caratterizzato da dislivelli molto consistenti: dagli oltre 4179 metri della Dent d'Hérens, il punto più elevato in corrispondenza della testata, la valle scende, in circa 32 chilometri in linea d'aria, agli $800 \mathrm{~m}$ circa dell'abitato di Roisan.

L'esame della carta geologica d'Italia (Foglio n 28 Aosta e Foglio n. 29 Monte Rosa) e la letteratura geologica (Baretti, 1893; Dal Piaz, 1992a, 1992b; Roda \& Zucali, 2008; Manzotti \& Ballèvre, 2014) permettono di delineare per la valle in oggetto il quadro litologico descritto qui di seguito. L'alta valle, tra la testata e l'abitato di Valpelline, appartiene alle falde austroalpine della Dent Blanche e del Mont Mary, costituite dalla Serie di Arolla (graniti, granodioriti e quarzodioriti di età permiana, trasformati in ortogneiss e scisti di vario tipo dall'orogenesi alpina) lungo le creste che delimitano la valle, dalla serie di Valpelline (kinzigiti e gneiss kinzigitici) nel settore assiale centrale (Fig. 3) e dagli affioramenti dell'Unità di Roisan (gneiss minuti e quarziti), marginalmente, lungo la sinistra orografica nei pressi dell'imbocco. Il Vallone di Ollomont è invece modellato nelle formazioni mesozoiche dei calcescisti e delle pietre verdi della Zona ofiolitica Ligure-Piemontese. Calcescisti e filladi, che

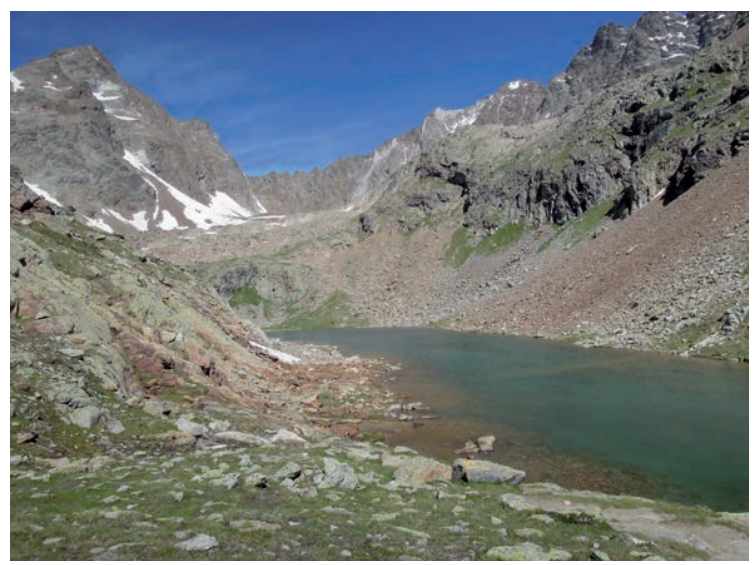

Fig. 3. La conca del Lac Long (18.VII.2014) modellata nel complesso kinzigitico della serie di Valpelline. La stazione è popolata da una carabidocenosi orofila della quale fanno parte Cicindela gallica, Nebria laticollis, N. jochischii, Oreonebria castanea, Pterostichus multipunctatus, Amara erratica, A. praetermissa, A. quenseli, Calathus melanocephalus e Cymindis vaporariorum. nel vallone sono il litotipo di gran lunga prevalente di tali formazioni, circondano un affioramento ofiolitico di limitata estensione, costituito da prasiniti e anfiboliti, localizzato nei dintorni dell'abitato di Glacier.

L'eterogeneità delle rocce affioranti nelle diverse zone del territorio in oggetto determina evidenti contrasti morfologici e pedologici. Nel Vallone di Ollomont, dominato alla testata dall'ampio circo glaciale della Conca di By, la presenza di rocce dalle caratteristiche meccaniche decisamente scadenti, soggette per di più ad alterazione chimica per dissoluzione dei carbonati (i calcescisti), e di affioramenti rocciosi relativamente poco tenaci (le ofioliti) ha dato origine a suoli più evoluti e a un paesaggio caratterizzato da pendii più dolci e arrotondati, colonizzati dalla prateria alpina sino a quote elevate. Nel tratto di valle a monte dell'abitato di Valpelline, invece, la compattezza e la tenacità dei litotipi dominanti sono testimoniate dalla morfologia aspra dei rilievi e dalla presenza diffusa di affioramenti rocciosi e di litosuoli (macereti a grandi blocchi e pietraie, esito di trasporto glaciale o della dinamica dei versanti). Il fondovalle ha conservato il tipico profilo a " $U$ " conferitogli dal glacialismo pleistocenico, mentre i valloni tributari, che sono infossati e dominati da creste rocciose e da pareti scoscese, si affacciano sul bacino principale attraverso un terrazzo sospeso (Fig. 4) -

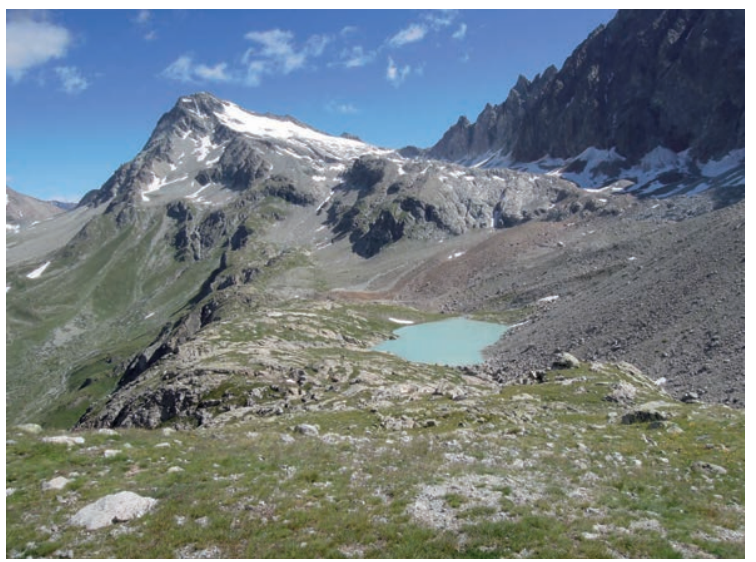

Fig. 4. Dossi montonati lungo il terrazzo sospeso dei Laghi del Morion nel Vallone di Ollomont (13.VIII.2013). Sullo sfondo a sinistra Il Col Fenêtre de Durand. Il terrazzo è popolato da Cicindela gallica, Carabus concolor, Nebria laticollis, Oreonebria castanea, O. picea, Bembidion bipunctatum, B. magellense, Amara erratica, A. quenseli e Cymindis vaporariorum. 
spesso costellato da dossi montonati e da massi erratici - in conseguenza di un'esarazione meno intensa operata dai rami glaciali laterali rispetto a quello principale. In alcuni valloni si osservano anche le morfologie tipiche del modellamento glaciale originate dall'ultima pulsazione nel corso della cosiddetta "Piccola Età Glaciale" (Fig. 5).

Il clima della Valpelline è tendenzialmente xerico. A causa della posizione geografica, infatti, il suo territorio, come si rileva in buona parte delle valli valdostane più interne, è poco esposto agli afflussi di aria umida di qualunque provenienza e fa parte della cosiddetta "isola di xericità intralpina" che copre un lungo tratto del fondovalle valdostano (cfr. Focarile, 1974, Mercalli, 2003). Lo si può desumere in tutta la sua evidenza dall'esame della carta delle isoiete e dalla consultazione del quadro delle precipitazioni medie annuali relative ad alcune stazioni pluviometriche della valle (Mercalli, 2003). Infatti, in corrispondenza dell'imbocco (agli $846 \mathrm{~m}$ della stazione di Roisan) si registra un media annua di precipitazioni di soli $607 \mathrm{~mm}$. Risalendo il fondovalle, i valori meteorici variano dai 650 $\mathrm{mm}$ di Valpelline (950 m) ai $703 \mathrm{~mm}$ di Ollomont (1337 $\mathrm{m})$, ai $598 \mathrm{~mm}$ di Oyace $(1367 \mathrm{~m})$ e agli $834 \mathrm{~mm}$ del Lago di Placemoulin (1968 m). Per quanto riguarda invece le dorsali che delimitano la valle, anche buona parte del versante sinistro, nonostante le quote elevate, usufruisce di apporti meteorici relativamente ridotti che si attestano tra i 700 e i $900 \mathrm{~mm}$; solo in corrispondenza del versante opposto e della testata i tassi medi sono relativamente più elevati, anche se, comunque, risultano per lunghi tratti inferiori ai $1400 \mathrm{~mm}$. Il regime pluviometrico (Mercalli, 2003) è molto regolare: tutte le stazioni sono caratterizzate da medie mensili poco variabili. Infatti, il divario tra il massimo assoluto (autunnale in tutte le stazioni) e il minimo assoluto (estivo nelle stazioni di Roisan, di Ollomont e del Lago di Placemoulin, invernale in quelle di Valpelline e Oyace) si aggira intorno a soli $30-50 \mathrm{~mm}$.

Dal punto di vista termico, il clima della valle è piuttosto rigido perché fortemente influenzato dai dislivelli, dall'esposizione tendenzialmente settentrionale del versante sinistro e dall'infossamento di lunghi tratti del fondovalle e di buona parte dei valloni secondari. Infatti, la carta delle isoterme (Mercalli, 2003) evidenzia temperature medie annue appena superiori a $8^{\circ} \mathrm{C}$ solo in corrispondenza della bassa valle tra Roisan e Valpelline. Risalendo il fondovalle, queste diminuiscono al di sotto dei $6^{\circ} \mathrm{C}$ già in corrispondenza di Oyace e al di sotto dei $4^{\circ} \mathrm{C}$ poco a monte di Bionaz. Nei valloni secondari, poi, le temperature medie annue scendono al di sotto di $0^{\circ} \mathrm{C}$ e, per lunghi tratti, al di sotto di $-5^{\circ} \mathrm{C}$. A causa del regime termico, quindi, le dorsali che delimitano la valle sono interessate da precipitazioni nevose per buona parte dell'anno (cfr. Carta della quantità media annua di neve fresca in Mercalli, 2003); inoltre, nelle zone più in quota $\mathrm{e}$ meno esposte, la copertura nevosa rimane al suolo dai 210 ai 365 giorni (cfr. Carta delle isochione in Mercalli, 2003) e va ad alimentare gli apparati glaciali che occupano la testata e parte dei due versanti.

Il bacino idrografico è perciò caratterizzato da un regime prevalentemente nivo-glaciale. Durante la stagione estiva, che è tendenzialmente siccitosa in buona parte del territorio (nelle cinque stazioni pluviometriche già citate, durante il trimestre giugno-luglio-agosto, si registrano valori complessivi di precipitazioni che non raggiungono i $150 \mathrm{~mm}$ ), le portate del torrente principale, dei suoi tributari e del fitto reticolo di torrentelli che discendono lungo i pendii sono comunque mantenute a un buon livello (soprattutto nei periodi più caldi) sia dalla graduale fusione della copertura nevosa, sia dall'ablazione. Pertanto,

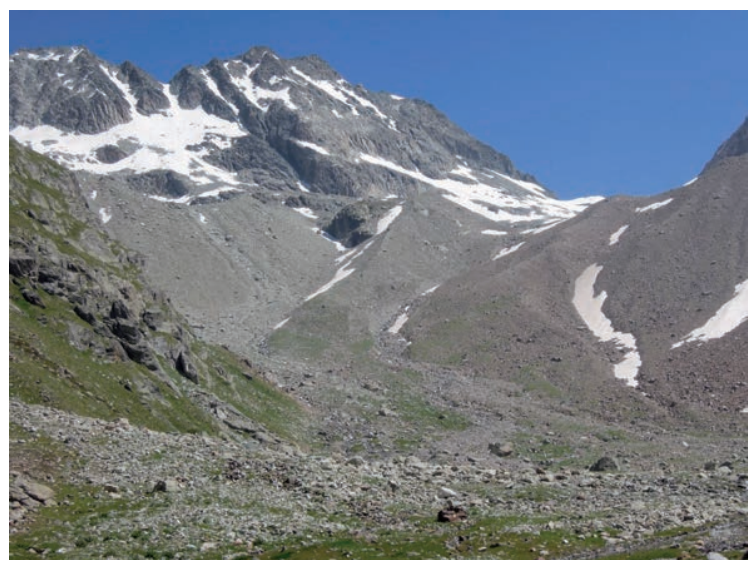

Fig. 5. Comba della Sassa (2.VIII.2013). Alla base della morena del ghiacciaio omonimo, prodotta dall'ultima pulsazione nel corso della cosiddetta "Piccola Età Glaciale", sono presenti popolazioni rispettivamente di Cicindela gallica, Carabus concolor, Nebria laticollis, $N$. rufescens, N. jockischii, Oreonebria castanea, Bembidion bipunctatum, B. geniculatum, B. magellense, Pterostichus multipunctatus, Amara erratica, A. quenseli, Calathus melanocephalus e Cymindis vaporariorum. 
gli afflussi idrici che ne derivano contribuiscono in larga parte a limitare le conseguenze della siccità estiva. Eventuali problemi di deficit idrico si possono forse verificare solo nel fondovalle principale lungo $\mathrm{i}$ pendii più esposti del versante destro (dove sono presenti alcune aree xerotermiche: cfr. Focarile, 1974).

Per quanto riguarda i consorzi forestali, dall'imbocco della valle si osserva la successione vegetazionale descitta qui di seguito. Nella fascia submontana - tra Roisan e Valpelline - predominano le latifoglie. Le formazioni boschive, infatti, sono costituite prevalentemente da consorzi misti Castanea-Fraxinus-Populus. Piccoli boschi a Quercus pubescens e/o a Pinus sylvestris occupano le zone più xeriche dell'imbocco. Nell'orizzonte montano è preponderante invece la presenza delle conifere. Estese peccete (Picea excelsa) occupano i tratti più ombrosi del fondovalle e del versante sinistro, mentre il versante opposto, più soleggiato, è colonizzato soprattutto da lariceti (Larix decidua). Si osservano ancora, più sporadici, consorzi misti di latifoglie nelle quali prevalgono il frassino (Fraxinus excelsior) e l'acero montano (Acer pseudoplatanus). Quest'ultima essenza è presente anche sia come boscaglie invadenti le formazioni aperte abbandonate, sia come vecchi alberi nei consorzi prativi ancora coltivati, fattore quest'ultimo che ha consentito di censire alcune importanti cenosi di specie di Drominii (v. Catalogo topografico).

Sopra il limite superiore della vegetazione forestale (Fig. 6), le specie arbustive che più di tutte ca-

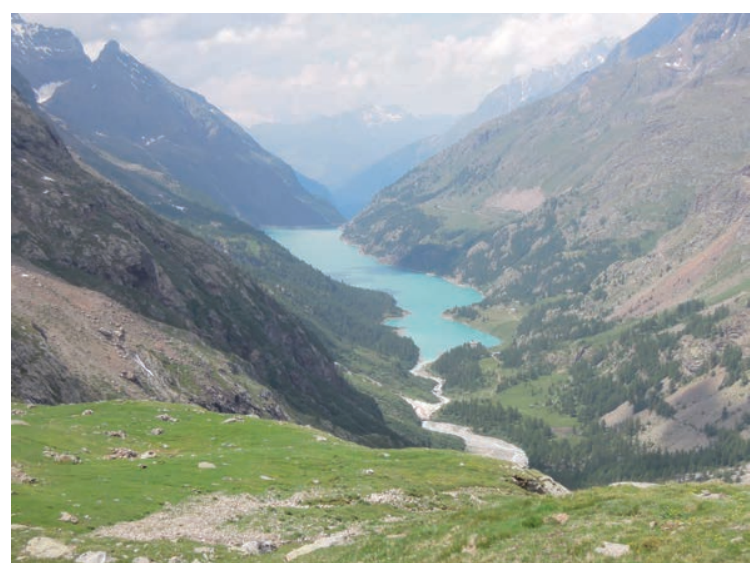

Fig. 6. L'alta Valpelline a monte del Lago di Placemoulin (20.VII.2013). Dal basso verso l'alto si può notare la successione vegetazionale (dalla fascia delle conifere alle falde detritiche d'alta quota) che caratterizza i due versanti. ratterizzano l'orizzonte subalpino sono l'ontano verde (Alnus viridis) (che occupa le aree più umide e fresche dei canaloni) e il rododendro (Rhododendron ferrugineum) (che colonizza invece i substrati più filtranti). Individui isolati di larice in facies arbustiva si osservano comunque sino a quote piuttosto elevate. I piani alpino e alto-alpino sono caratterizzati da un'estesa fascia di pascoli caratterizzati da ampie soluzioni di continuità per la presenza di affioramenti rocciosi, di macereti a grandi blocchi o di pietraie, substrati privi di vegetazione o occupati solo sporadicamente da chiazze di fitocenosi pioniere.

\section{CAtalogo topografico}

Per la nomenclatura di quasi tutte le specie e per l'attribuzione del corotipo di competenza a ciascuna di esse, si è fatto riferimento alla Checklist dei Carabidi italiani elaborata da Vigna Taglianti (2005), apportando peraltro alcune variazioni che sono di volta in volta giustificate con nota a piè di pagina. Invece, per quanto riguarda la nomenclatura dei Bembidiini, si è presa come base la Checklist nel contributo più recente di Neri et al. (2011). Sono segnalate con un asterisco le specie che, pur già note dell'arco alpino occidentale, non lo erano delle Alpi Pennine. Per ogni località segnalata è indicata la citazione bibliografica o il raccoglitore (LB=Luigi Bisio).

\section{Cicindela (Cicindela) campestris campestris Linné, 1758}

«Valpelline: chemin d'Ollomont» (Henry, 1915, 1916); «De Valpelline à Ollomont» (Pavesi, 1904); Ollomont-Col de Fenêtre (Born, 1900b); Les Crêtes (Ollomont) (Henry, 1938); Conca di By (Ollomont) (Henry, 1938); ibidem a m 2000 (LB); Lacs de Thoules (Conca di By, Ollomont) $m$ 22502350 (LB); Comba di Berrio (Ollomont) (Henry, 1938). Corotipo: Paleartico (PAL).

\section{Cicindela (Cicindela) sylvicola Dejean in La-} treille \& Dejean, 1822

«Du Col de Fenêtre à Aoste» (Gaud, 1902; Pavesi, 1904); Prelé (Oyace) m 1100 (LB).

Corotipo: Centroeuropeo (CEU).

\section{Cicindela (Cicindela) gallica Brullé, 1834}

Valpelline (Bischoff-Ehinger \& Stierlin, 1870: sub alpestris; Magistretti, 1965); Champillon (Ollomont) (Henry, 1935); Col Champillon (Ollomont) m 2700 (LB); Conca di By (Ollomont) (Henry, 1935); Bivacco Savoie (Conca di By, Ollomont) $\mathrm{m}$ 2700 (LB); Lacs de Thoules (Conca di By, Ollomont) $m$ 2250- 
2350 (LB); «Du Col de Fenêtre à Aoste» (Gaud, 1902: sub chloris; Pavesi, 1904); Les Crêtes (Ollomont) (Henry, 1938); Laghi del Morion (Glacier, Ollomont) m 2500 (LB); Comba di Berrio (Ollomont) (Henry, 1938); Plan Meulet (Valpelline) (Henry, 1938); Rifugio Crête Seche (Bionaz) m 2000 (LB); Comba della Sassa (Bionaz) m 2500-2600 (LB); Lac Long (Lago di Placemoulin, Bionaz) m 2720 (LB); Comba Braoulé (Bionaz) m 2300 (Henry, 1915: sub chloris); Alpage Pra de Dieu (Comba d'Arbiére, Bionaz) m 2300 (LB); Comba di Verdzignola (Valpelline) (Henry, 1938); Comba d'Arpisson (Valpelline) (Henry, 1938); Viou (Roisan) (Henry, 1935).

Corotipo: Centroeuropeo (CEU).

\section{Cicindela (Cicindela) hybrida transversalis De-} jean in Latreille \& Dejean, 1822

Souhairoux (Valpelline) (Henry, 1915: sub hybrida var. riparia); «De Valpelline à Ollomont» (Pavesi, 1904: sub hybrida var. riparia); Ollomont-Col de Fenêtre (Born, 1900b: sub hybrida var. riparia).

Corotipo: Sibirico-Europeo (SIE).

\section{Carabus (Archicarabus) nemoralis nemoralis} O.F. Müller, 1764

Valpelline (Pavesi, 1904; Henry, 1915); Doues (Casale et al., 1982); ibidem a m 1100 (LB); Ollomont (Casale et al., 1982); Les Crêtes (Ollomont) (Henry, 1938); Rey (Ollomont) (Henry, 1915); Glacier (Ollomont) m 1500-1600 (LB); Thoules (Casale et al., 2006); ibidem a m 1050 (LB); Colle di Saint-Barthélemy-Oyace (Born, 1900a); Colle di Saint-Barthélemy (Born, 1904); Prelé (Oyace) m 1100 (LB); Bionaz m 1700 (LB); Comba d'Arpisson (Valpelline) (Henry, 1938). Corotipo: Europeo (EUR).

\section{Carabus (Mesocarabus) problematicus proble- maticus Herbst, 1786}

Les Crêtes (Ollomont) (Henry, 1938: erroneamente sub «Carabus catenulatus Scop.»).

La segnalazione della presenza in Valpelline di C. catenulatus Scopoli, 1763 da parte di Henry (1938) è palesemente errata. Sicuramente egli voleva riferirsi a $C$. problematicus ( $=C$. catenulatus Fabricius, $1792 ;=C$. catenulatus Duftschmid, 1812) (nec catenulatus Scopoli, 1763), entità ampiamente diffusa in Valle d'Aosta.

CoRotipo: Europeo (EUR).

\section{Carabus (Orinocarabus) concolor Fabricius,} 1792

Valpelline (Pavesi, 1904: sub Orinocarabus alpestris var. bernhardinus); Ollomont-Col de Fenêtre (Born, 1900b: sub Carabus var. bernhardinus); Conca di By (Ollomont) (Bischoff-Ehinger \& Stierlin, 1870: sub alpinus; Henry, 1915: sub alpinus); Bivacco Savoie (Conca di By, Ollomont) m 2700
(LB); Lacs de Thoules (Conca di By, Ollomont) m 2250-2350 (LB); Col Fenêtre (Breuning, 1932-1936: sub concolor bernhardinus); Laghi del Morion (Glacier, Ollomont) m 2500 (Bisio, 2002); Pian Meulet (Valpelline) (Henry, 1938: sub concolor bernhardinus); Comba della Sassa (Bionaz) m 25002600 (LB); Lac de Mont Ros (Lago di Placemoulin, Bionaz) m 2700 (Bisio, 2002); Rifugio Collon (Comba d'Oren, Lago di Placemoulin, Bionaz) m 2700 (Bisio, 2002); Prarayer (Bionaz) m 2000 (Henry, 1915; sub Orinocarabus alpinus); Alpage Bella Tza (Prarayer, Bionaz) m 2747 (LB); Comba di Livournea (Bionaz) m 2000 (Henry, 1915: sub Carabus alpinus); Bivacco Pra de Dieu (Comba d'Arbiére, Bionaz) m 2530 (LB); Colle di Saint-Barthélemy-Oyace (Born, 1900a); Colle di Saint-Barthélemy (Pavesi, 1904; Born, 1904); Comba d'Arpisson (Valpelline) (Henry, 1938: sub concolor bernhardinus); Viou (Roisan) (Henry, 1935: sub concolor v. bernhardinus). Corotipo: W-Alpino (ALPW).

\section{Carabus (Chaetocarabus) intricatus Linné, 1761}

Rhins (Roisan) m 900 (LB); Valpelline m 960 (LB); Doues m 1100 (LB); Chez Collet (Ollomont) m 1280 (LB); Thoules m 1050 (LB); Prelé (Oyace) m 1100 (LB);

Corotipo: Europeo (EUR).

\section{Carabus (Platycarabus) depressus depressus Bonelli, 1810}

Valpelline (Pavesi, 1904: sub Plectes depressus; Casale et al., 2006); Souhairoux (Valpelline) (Henry, 1915); Chez Collet (Ollomont) m 1280 (LB); Glacier (Ollomont) m 1500-1600 (LB); Conca di By (Ollomont) (Bischoff-Ehinger \& Stierlin, 1870; Henry, 1938: sub depressus v. jurinei); Bionaz m 1700 (LB); Alpage Bella Tza (Prarayer, Bionaz) m 2747 (LB); Colle di Saint-Barthélemy-Oyace (Born, 1900a); Colle di Saint-Barthélemy (Born, 1904); Prelé (Oyace) m 1100 (LB); Comba d'Arpisson (Valpelline) (Henry, 1935: sub depressus v. jurinei).

Corotipo: Centroeuropeo (CEU).

\section{Carabus (Megodontus) germarii fiorii Born,} 1901

«Valpelline: chemin de Semon» (Henry, 1915: sub violaceus); Rey (Ollomont) (Henry, 1915: sub violaceus).

Corotipo: S-Europeo (SEU).

11. Leistus (Leistus) ferrugineus (Linné, 1758)

«Valpelline: chemin de Doues» (Henry, 1915).

Oltre che della valle in oggetto, in Valle d'Aosta L. ferrugineus è noto della valle di Champorcher (Bisio, dati inediti), della Val d'Ayas (Bisio, 2006) e della Valle di Saint-Barthélemy (Bisio, 2013b).

Corotipo: Europeo (EUR). 
12. Leistus (Pogonophorus) spinibarbis spinibarbis (Fabricius, 1775)

«Valpelline: chemin d'Ollomont» (Henry, 1915); Prémont (Bionaz) m 1800 (Henry, 1915).

In Valle d'Aosta L. spinibarbis è noto ancha della Valle di Cogne (Bisio, dati inediti).

COROTIPO: Europeo (EUR).

13. Nebria (Nebriola) laticollis Dejean, 1826

Lacs de Thoules (Conca di By, Ollomont) m 2250-2350 (LB); Lago Fenêtre (Conca di By, Ollomont) m 2750 (LB); Col Fenêtre (Pavesi, 1904); «Du Col Fenêtre à Aoste» (Gaud, 1902); Laghi del Morion (Glacier, Ollomont) m 2500 (Bisio, 1999); Bivacco Spataro (Plan de la Sabbla, Bionaz) m 2600 (LB); Comba della Sassa (Bionaz) m 2500-2600 (LB); Lac Long (Lago di Placemoulin, Bionaz) m 2720 (LB); Rifugio Collon (Comba d'Oren, Lago di Place Moulin, Bionaz) m 2700 (Bisio, 1999).

Corotipo: W-Alpino (ALPW).

14. Nebria (Boreonebria) rufescens (Stroem, 1768) Valpelline (Bischoff-Ehinger \& Stierlin, 1870: sub gyllenhali); Pavesi, 1904: sub gyllenhali; Henry, 1915: sub gyllenhali); Chez Collet (Ollomont) m 1280 (LB); Col Fenêtre (Pavesi, 1904: sub gyllenhali;); «Du Col Fenêtre à Aoste» (Gaud, 1902: sub gyllenhali); Les Crêtes (Ollomont) (Henry, 1935: sub gyllenhali; 1938: sub gyllenhali); Thoules m 1100 (Ravizza, 1972: sub gyllenhali); Bionaz (Henry, 1915: sub gyllenhali); Comba della Sassa (Bionaz) m 2500-2600 (LB); Prarayer (Henry, 1915: sub gyllenhali); Colle di Saint-Barthélemy-Oyace (Born, 1900b: sub gyllenhali); Comba d'Arpisson (Valpelline) (Henry, 1935: sub gyllenhali).

Corotipo: Oloartico (OLA).

15. Nebria (Eunebria) jockischii Sturm, 1815

Valpelline (Bischoff-Ehinger \& Stierlin, 1870; Pavesi, 1904); Chez Collet (Ollomont) m 1280 (LB); «Du Col Fenêtre à Aoste» (Gaud, 1902); Rifugio Crête Seche (Bionaz) m 2300 (LB); Comba della Sassa (Bionaz) m 2500-2600 (LB); Lac Long (Lago di Placemoulin, Bionaz) m 2720 (LB); Comba Braoulé (Bionaz) m 2300 (Henry, 1915). Corotipo: Centroeuropeo (CEU).

16. Nebria (Eunebria) picicornis picicornis (Fabricius, 1792)

Valpelline m 960 (LB); Prelé (Oyace) m 1100 (LB); Comba d'Arpisson (Valpelline) (Henry, 1938).

COROTIPO: Europeo (EUR).

\section{Oreonebria (Oreonebria) castanea castanea (Bonelli, 1810) \\ Valpelline (Bischoff-Ehinger \& Stierlin, 1870: sub Nebria;}

Pavesi, 1904: sub Nebria); Ollomont (Pavesi, 1904: sub Nebria); Ollomont-Col de Fenêtre (Born, 1900b: sub Nebria); «Du Col de Fenêtre à Aoste» (Gaud, 1902: sub Nebria); Les Crêtes (Ollomont) (Henry, 1938: sub Nebria); Conca di By (Ollomont) m 2000 (Henry, 1915: sub Nebria, 1938: sub Nebria); Monte Chénaille (Conca di By, Ollomont) m 2400 (Focarile \& Casale, 1978: sub Nebria); Bivacco Savoie (Conca di By, Ollomont) m 2700 (Bisio, 2008); Lacs de Thoules (Conca di By, Ollomont) m 2250-2350 (LB); Lago Fenêtre (Conca di By, Ollomont) m 2750 (Bisio, 2008); Laghi del Morion (Glacier, Ollomont) m 2550 (Bisio, 2008); Bivacco Spataro (Plan de la Sabbla, Bionaz) m 2600 (Bisio, 2008); Comba della Sassa (Bionaz) m 2500-2600 (LB); Lac Long (Lago di Placemoulin, Bionaz) m 2720 (LB); Lago Morto (Lago di Placemoulin, Bionaz) m 2800 (Bisio, 2008); Rifugio Collon (Comba d'Oren, Lago di Placemoulin, Bionaz) m 2700 (Bisio, 2008); Comba Braoulé (Bionaz) m 2000-2300 (Henry, 1915: sub Nebria); Prarayer (Lago di Placemoulin, Bionaz) m 2300 (Focarile \& Casale, 1978: sub Nebria); Alpage Bella Tza (Prarayer, Bionaz) m 2747 (LB); Colle Livournea (Lago di Placemoulin, Bionaz) m 2800 (Bisio, 2008); Comba d'Arbiére (Bionaz) (Henry, 1915: sub Nebria); Becca d'Aquelou (Bionaz) m 2300 (Focarile \& Casale, 1978: sub Nebria); Colle di Saint-Barthélemy-Oyace (Born, 1900b); Comba d'Arpisson (Valpelline ) (Henry, 1935: sub Nebria); Comba di Valfreyde (Valpelline) (Henry, 1935: sub Nebria).

Corotipo: Centroeuropeo (CEU).

\section{Oreonebria (Oreonebria) picea picea (Dejean,} 1826)

Conca di By (Ollomont) (Henry, 1915: sub Nebria); Monte Chénaille (Conca di By) (Focarile \& Casale, 1978: sub $\mathrm{Ne}$ bria); Laghi del Morion (Glacier, Ollomont) m 2500 (LB); Lago d'Incliousa (Monte Morion, Glacier, Ollomont) m 2400 (Bisio, 2008); Prarayer (Lago di Placemoulin, Bionaz) m 2300 (Focarile \& Casale, 1978: sub Nebria); Alpage Bella Tza (Prarayer, Bionaz) m 2747 (LB); Becca d'Aquelou (Bionaz) m 2300 (Focarile \& Casale, 1978: sub Nebria). COROTIPO: S-Alpino (ALPS).

19. Notiophilus aestuans Dejean, 1826 Oyace m 1350, 1 es. 24.VI.2013 (LB). COROTIPO: Europeo (EUR).

20. Notiophilus palustris (Duftschmid, 1812) Rhins (Roisan) m 900 (LB); Doues m 1100 (LB); Valpelline m 960 (LB); Thoules m 1050 (LB); Prarayer (Bionaz) m 2000 (Henry, 1915).

CorotiPo: Sibirico-Europeo (SIE).

21. Notiophilus biguttatus (Fabricius, 1779)

Prelé (Oyace) m 1100 (LB).

COROTIPO: Oloartico (OLA). 


\section{Loricera pilicornis (Fabricius, 1775)}

Chez Collet (Ollomont) m 1280, 1 es. 7.IV.2014 (LB).

Le popolazioni valdostane di L. pilicornis sono di probabile origine transalpina (Casale \& Vigna Taglianti, 1993). Ritenuta per lungo tempo esclusiva delle valli valdostane più occidentali (Magistretti, 1965; Allegro et al., 2011; Bisio \& Giuntelli, 2014), questa entità, oltre che nella valle in oggetto, è stata rinvenuta recentemente anche in Valtournenche (Bisio et al., in stampa) e nella Valle di Saint-Barthélemy (Bisio, 2013b). Corotipo: Oloartico (OLA).

23. Trechus (Trechus) quadristriatus (Schrank, 1781) Prelé (Oyace) m 1100 (LB); Bionaz m 1700 (LB); La Ferrere (Bionaz) m 1650 (LB).

Corotipo: Turanico-Europeo-Mediterraneo (TEM).

24. Tachyura (Tachyura) sexstriata (Duftschmid, 1812) Prelé (Oyace) m 1100 (LB).

Corotipo: Europeo (EUR).

25. Asaphidion caraboides (Scrank, 1781)

Thoules m 1100 (Ravizza, 1972).

Corotipo: S-Europeo (SEU).

26. Asaphidion pallipes (Duftschmid, 1812)

Thoules m 1100 (Ravizza, 1972).

Corotipo: Sibirico-Europeo (SIE).

27. Bembidion (Chlorodium) pygmaeum (Fabricius, 1792)

Thoules m 1100 (Ravizza, 1972).

Corotipo: Europeo (EUR).

28. Bembidion (Metallina) lampros (Herbst, 1784) Rhins (Roisan) m 900 (LB); Doues m 1100 (LB); Valpelline (Henry, 1935, 1938); ibidem a m 960 (LB); Champillon (Ollomont) (Henry, 1935); Thoules m 1050 (LB); Prelé (Oyace) m 1100 (LB); Oyace m 1350-1450 (LB); Bionaz m 1700 (LB); La Ferrere (Bionaz) m 1650 (LB); Comba Braoulé (Bionaz) m 2300 (Henry, 1915: sub Bembidium).

Corotipo: Paleartico (PAL).

29. Bembidion (Emphanes) azurescens Dalla Torre, 1877

Thoules m 1100 (Ravizza, 1972).

Corotipo: Europeo (EUR).
30. Bembidion (Bembidion) quadrimaculatum (Linné, 1761)

Doues m 1100 (LB); Prelé (Oyace) m 1100 (LB).

Corotipo: Oloartico (OLA).

31. Bembidion (Princidium) punctulatum punctulatum Drapiez, 1821

Valpelline m 960 (LB).

CorotiPo: Centroasiatico-Europeo-Mediterraneo (CEM).

32. Bembidion (Testedium) bipunctatum bipunctatum (Linné, 1761) ${ }^{2}$

Valpelline (Bischoff-Ehinger \& Stierlin, 1870); «De Valpelline à Ollomont» (Pavesi, 1904); Ollomont-Col de Fenêtre (Born, 1900b: sub Bembidium); Bivacco Savoie (Conca di By, Ollomont) m 2700 (Bisio, 2009a: sub Ocydromus); Lacs de Thoules (Conca di By, Ollomont) m 2250-2350 (LB); Lago Fenêtre (Conca di By, Ollomont) m 2750 (Bisio, 2009a); Laghi del Morion (Glacier, Ollomont) m 2500 (Bisio, 2009a: sub Ocydromus); Bivacco Spataro (Plan de la Sabbla, Bionaz) m 2600 (Bisio, 2009a: sub Ocydromus); Berrier (Bionaz) m 2300 (Henry, 1915: sub Bembidium); Comba della Sassa (Bionaz) m 2500-2600 (LB); Prarayer (Bionaz) (Henry, 1915: sub Bembidium, 1935); Colle di Vessona (Oyace) m 2783 (LB). CoRotipo: W-Paleartico (WPA). ${ }^{3}$

33. Bembidion (Bembidionetolitzkya) varicolor (Fabricius, 1803)

Rhins (Roisan) m 900 (LB); Valpelline m 800 (Ravizza, 1972: sub tricolor); ibidem a m 960 (LB); Glacier (Ollomont) m 1550 (Ravizza, 1972: sub tricolor); Thoules m 1100 (Ravizza, 1972: sub tricolor); Prelé (Oyace) m 1100 (LB).

Corotipo: Europeo (EUR).

34. Bembidion (Bembidionetolitzkya) fasciolatum (Duftschmid, 1812)

Thoules m 1100 (Ravizza, 1972).

Corotipo: Centroeuropeo (CEU).

35. Bembidion (Bembidionetolitzkya) ascendens K. Daniel, 1902

Valpelline m 960 (LB).

Corotipo: Centroeuropeo (CEU).

36. Bembidion (Bembidionetolitzkya) complanatum Heer, 1837
Sensu Neri et al. (2013).

Sensu Vigna Taglianti (com. pers., 2011). 
Rhins (Roisan) m 900 (LB); Valpelline m 800 (Ravizza, 1972); ibidem a m 960 (LB); Glacier (Ollomont) m 1550 (Ravizza, 1972); Thoules m 1100 (Ravizza, 1972).

Corotipo: Centroeuropeo (CEU).

\section{Bembidion (Bembidionetolitzkya) longipes K.} Daniel, 1902

Glacier (Ollomont) m 1550, 2 es. 8.VII.1969 (Ravizza, 1972); Place Moulin m 1850, 1 es. 2.VI.1968 (Ravizza, 1972).

Corotipo: Centroeuropeo (CEU).

\section{Bembidion (Bembidionetolitzkya) geniculatum} geniculatum Heer, 1837

Rhins (Roisan) m 900 (LB); Valpelline m 960 (LB); Chez Collet (Ollomont) m 1280 (LB); Lacs de Thoules (Conca di By, Ollomont) m 2250-2350 (LB); Glacier (Ollomont) m 1550 (Ravizza, 1972: sub Bembidion); Comba della Sassa (Bionaz) m 2500-2600 (LB); Place Moulin (Bionaz) m 1850 (Ravizza, 1972: sub Bembidion); Comba·di Valfreyde (Valpelline) (Henry, 1935 sub redtenbacheri).

Corotipo: Europeo (EUR).

39. Bembidion (Bembidionetolitzkya) tibiale (Duftschmid, 1812)

Rhins (Roisan) m 900 (LB); «Valpelline: chemin de Doues» (Henry, 1915: sub Bembidium); Valpelline m 800 (Ravizza, 1972); ibidem a m 960 (LB); Chez Collet (Ollomont) m 1280 (LB); Champillon (Ollomont) (Henry, 1938); Conca di By (Ollomont) (Henry, 1938); Thoules m 1100 (Ravizza, 1972); ibidem a m 1050 (LB); Place Moulin (Bionaz) m 1850 (Ravizza, 1972).

Corotipo: Europeo (EUR).

40. Bembidion (Nepha) genei illigeri Netolitzky, 1914

Doues m 1100 (LB).

CoRotipo: Europeo-Mediterraneo (EUM).

\section{Bembidion (Testediolum) pyrenaeum poenini} Marggi \& Huber, 1993

Col Champillon (Ollomont) m 2800 (Bisio, 2009a: sub Ocydromus); Conca di By (Ollomont) (Henry, 1938); Lacs de Thoules (Conca di By, Ollomont) m 2250-2350 (LB); «De Valpelline à la Balme d'Ollomont» (Pavesi, 1904: sub salhbergii var. rhaeticum).

Corotipo: S-Europeo (SEU).

42. Bembidion (Testediolum) glaciale Heer, 1837 Ollomont-Col de Fenêtre (Born, 1900b: sub Bembidium); «De Valpelline à la Balme d'Ollomont» (Pavesi, 1904: sub salhbergii var. glaciale); Lacs de Thoules (Conca di By, Ol- lomont) m 2250-2350 (LB); Comba·di Valfreyde (Valpelline) (Henry, 1935).

Corotipo: Centroeuropeo (CEU).

\section{Bembidion (Testediolum) magellense alpicola} (Jeannel, 1941)

Col Champillon (Ollomont) m 2800 (Bisio, 2009a: sub Ocydromus); Bivacco Savoie (Conca di By, Ollomont) m 2700 (Bisio, 2009a: sub Ocydromus); Lacs de Thoules (Conca di By, Ollomont) m 2313 (Bisio, 2009a: sub Ocydromus); Lago Fenêtre (Conca di By, Ollomont) m 2750 (Bisio, 2009a: sub Ocydromus); Laghi del Morion (Glacier, Ollomont) m 2500 (Bisio, 2009a: sub Ocydromus); Bivacco Spataro (Plan de la Sabbla, Bionaz) m 2600 (Bisio, 2009a: sub Ocydromus); Comba della Sassa (Bionaz) m 2500-2600 (LB); Ghiacciaio della Sassa (Bionaz) m 3000 (Bisio, 2009a: sub Ocydromus); Lac Mort (Lago di Placemoulin, Bionaz) m 2700 (Bisio, 2009a: sub Ocydromus); Rifugio Collon (Comba d'Oren, Lago di Placemoulin, Bionaz) m 2700 (Bisio, 2009a: sub Ocydromus); Colle Livournea (Lago di Placemoulin, Bionaz) m 2800 (LB); Colle di Vessona (Oyace) m 2783 (LB).

Corotipo: Alpino-Appenninico (ALAP).

44. Bembidion (Ocydromus) decorum decorum (Zenker in Panzer, 1799)

Valpelline m 800 (Ravizza, 1972); ibidem a m 960 (LB); Glacier (Ollomont) m 1550 (Ravizza, 1972: sub Bembidion); Thoules m 1100 (Ravizza, 1972).

Corotipo: Centroasiatico-Europeo (CAE).

45. Bembidion (Peryphus) femoratum Sturm, 1825 Prelé (Oyace) m 1100 (LB).

Corotipo: Sibirico-Europeo (SIE).

46. Bembidion (Peryphus) tetracolum Say, 1823 Valpelline (Henry, 1935: sub ustulatum); Valpelline m 800 (Ravizza, 1972: sub ustulatum); Glacier (Ollomont) m 1550 (Ravizza, 1972: sub ustulatum); ibidem a 1500-1600 (LB); Thoules m 1100 (Ravizza, 1972: sub ustulatum); ibidem a m 1050 (LB); Prelé (Oyace) m 1100 (LB); La Ferrere (Bionaz) m 1650 (LB).

Corotipo: Paleartico (PAL).

47. Bembidion (Peryphus) bualei du Val, 1852

Rhins (Roisan) m 900 (LB); Valpelline m 800 (Ravizza, 1972: sub andreae bualei); Glacier (Ollomont) m 1550 (Ravizza, 1972: sub andreae bualei); Thoules m 1100 (Ravizza, 1972: sub andreae bualei); Prelé (Oyace) m 1100 (LB); Placemoulin m 1850 (Ravizza, 1972: sub andreae bualei).

Corotipo: Europeo (EUR). 
48. Bembidion (Peryphus) incognitum G. Müller, 1931

Lacs de Thoules (Conca di By, Ollomont) m 2250-2350 (LB); Alpage Bella Tza (Prarayer, Bionaz) m 2747 (LB); Berrier (Bionaz) (Henry, 1915: sub Bembidium alpinum); Comba Braoulé (Bionaz) m 2300 (Henry, 1915: sub Bembidium alpinum).

CoRotipo: Centroeuropeo (CEU).

49. Bembidion (Peryphanes) deletum Audinet-Serville, 1821

Doues m 1000 (LB); Valpelline 1500 (Henry, 1938: sub Bembidium nitidulum); (Henry, 1938: sub Bembidium nitidulum); Comba di Valfreyde (Valpelline) m 1500 (Henry, 1916: sub Bembidium nitidulum).

COROTIPO: Europeo (EUR).

*50 Bembidion (Peryphanes) pallidicorne G. Müller, 1921

Prelé (Oyace) m 1100 (LB).

Specie nuova per la Valle d'Aosta e per le Alpi Pennine.

Corotipo: W-Alpino (ALPW).

51. Sinechostictus (Sinechostictus) ruficornis (Sturm, 1825)

Rhins (Roisan) m 900 (LB); Valpelline m 800 (Ravizza, 1972: sub Bembidion); ibidem a m 960 (LB); Glacier (Ollomont) m 1550 (Ravizza, 1972: sub Bembidion); Thoules m 1100 (Ravizza, 1972: sub Bembidion); ibidem a m 1050 (LB); Place Moulin m 1850 (Ravizza, 1972: sub Bembidion).

Corotipo: Centroeuropeo (CEU).

52. Sinechostictus (Sinechostictus) decoratus (Duftschmid, 1812)

Thoules m 1050 (LB); Place Moulin m 1850 (Ravizza, 1972: sub Bembidion).

Corotipo: Centroeuropeo (CEU).

53. Sinechostictus elongatus (Dejean, 1831)

«Valpelline: chemin d'Ollomont» (Henry, 1916: sub Bembidium).

Forse questa segnalazione dovrebbe essere confermata, poichè la specie non è mai più stata ritrovata, né in Valpelline, né in altre valli valdostane.

Corotipo: S-Europeo (SEU).

54. Sinechostictus (Pseudolimnaeum) doderoi (Ganglbauer, 1891)

Valpelline m 800, 1 es. 11.IX.1969 (Ravizza, 1972: sub Bembidion).

Corotipo: Centroeuropeo (CEU).
55. Stomis (Stomis) pumicatus (Panzer, 1796)

Chez Collet (Ollomont) m 1280, 1 es. 29.IV.2014 (LB).

COROTIPO: Europeo (EUR).

56. Poecilus (Poecilus) cupreus (Linné, 1758)

Rhins (Roisan) m 900 (LB); Doues m 1100 (LB); «Valpelline: chemin d'Ollomont» (Henry, 1915); Mont Durand (By, Ollomont) (Henry, 1938); Thoules m 1050 (LB); Prelé (Oyace) m 1100 (LB); Comba d'Arpisson (Valpelline) (Henry, 1938); Comba di Valfreyde (Valpelline) (Henry, 1935).

Corotipo: Asiatico-Europeo (ASE).

57. Poecilus (Poecilus) versicolor (Sturm, 1824)

Doues m 1100 (LB); Chez Collet (Ollomont) m 1280 (LB); Glacier (Ollomont) m 1500-1600 (LB); Thoules m 1050 (LB); Prelé (Oyace) m 1100 (LB); Bionaz m 1700 (LB); La Ferrere (Bionaz) m 1650 (LB); Comba d'Arpisson (Valpelline) (Henry, 1938: sub coerulescens).

COROTIPO: Asiatico-Europeo (ASE).

58. Poecilus (Macropoecilus) lepidus gressorius (Dejean, 1828)

Rhins (Roisan) m 900 (LB); Doues m 1100 (LB); Valpelline (Henry, 1915); Chez Collet (Ollomont) m 1280 (LB); Rey (Ollomont) (Henry, 1915); Glacier (Ollomont) m 1500-1600 (LB); Conca di By (Ollomont) (Henry, 1935); Thoules m 1050 (LB); Prelé (Oyace) m 1100 (LB); Bionaz (Henry, 1935); ibidem a m 1700 (LB); La Ferrere (Bionaz) m 1650 (LB).

CoRotipo: Sibirico-Europeo (SIE).

59. Pterostichus (Phonias) strenuus (Panzer, 1796) Doues m 1100 (LB); Glacier (Ollomont) m 1500-1600 (LB); Thoules m 1050 (LB); La Ferrere (Bionaz) m 1650 (LB). COROTIPO: Asiatico-Europeo (ASE).

60. Pterostichus (Bothriopterus) oblongopunctatus (Fabricius, 1787)

Doues m 1100 (LB); Valpelline (Henry, 1915; Schatzmayr, 1930); Chez Collet (Ollomont) m 1280 (LB); Glacier (Ollomont) m 1500-1600 (LB); Thoules m 1050 (LB); Prelé (Oyace) m 1100 (LB); La Ferrere (Bionaz) m 1650 (LB). Corotipo: Asiatico-Europeo (ASE).

61. Pterostichus (Platysma) niger (Schaller, 1783) Rhins (Roisan) m 900 (LB); Doues m 1000-1100 (LB); «Valpelline: chemin d'Ollomont» (Henry, 1915); Chez Collet (Ollomont) m 1280 (LB); Oyace m 1350-1450 (LB); Bionaz $\mathrm{m} 1700$ (LB).

Corotipo: Asiatico-Europeo (ASE).

62. Pterostichus (Morphnosoma) melanarius (Illiger, 1798) 
Doues m 1100 (LB); Valpelline (Pavesi, 1904: sub vulgaris; Schatzmayr, 1930: sub vulgaris); "Valpelline: chemin de Doues et d'Ollomont» (Henry, 1915: sub vulgaris); Champillon (Ollomont) (Henry, 1935: sub Omaseus vulgaris); Glacier (Ollomont) m 1500-1600 (LB); Les Crêtes (Ollomont) (Henry, 1938: sub vulgaris); Conca di By (Ollomont) (Henry, 1915: sub vulgaris; 1935: sub Omaseus vulgaris); Colle di Saint-Barthélemy-Oyace (Born, 1900b: sub vulgaris); Comba d'Arpisson (Valpelline) (Henry, 1938).

Corotipo: Oloartico (OLA).

63. Pterostichus (Pseudomaseus) nigrita (Paykull, 1790)

Valpelline (Henry, 1935).

Questa segnalazione potrebbe riferirsi alla specie seguente. Corotipo: Paleartico (PAL).

\section{Pterostichus (Pseudomaseus) rhaeticus Heer,} 1837

Doues m 1100 (LB).

Corotipo: Europeo (EUR).

65. Pterostichus (Oreophilus) multipunctatus (Dejean, 1828)

Valpelline (Pavesi, 1904); Ollomont-Col de Fenêtre (Born, 1900b); Ollomont (Pavesi, 1904); «du Col de Fenêtre à Aoste» (Gaud, 1902: sub Feronia); Champillon (Ollomont) (Henry, 1935); Glacier (Ollomont) m 1800 (LB); Les Crêtes (Ollomont) (Henry, 1938); Conca di By (Ollomont) (Bischoff-Ehinger \& Stierlin, 1870; Henry, 1915); Mont Durand (Conca di By, Ollomont) (Henry, 1938); Lacs de Thoules (Conca di By, Ollomont) m 2250-2350 (LB); Plan Meulet (Valpelline) (Henry, 1938); Comba della Sassa (Bionaz) $m$ 2500-2600 (LB); Lac Long (Lago di Placemoulin, Bionaz) m 2720 (LB); Rifugio Collon (Comba d'Oren, Lago di Place Moulin) m 2500 (LB); Comba Braoulé (Bionaz) (Henry, 1915); Alpage Bella Tza (Prarayer, Bionaz) m 2747 (LB); Comba di Livournea (Bionaz) (Henry, 1915); Comba d'Arbiére (Bionaz) (Henry, 1915); Bivacco Pra de Dieu (Comba d'Arbiére, Bionaz) m 2400-2530 (LB); Colle di Saint-Barthélemy-Oyace (Born, 1900b); Comba di Verdzignola (Valpelline) (Henry, 1938); Comba d'Arpisson (Valpelline) (Henry, 1938); Comba di Valfreyde (Valpelline) (Henry, 1935).

Corotipo: S-Alpino (ALPS).

66. Abax (Abax) parallelepipedus parallelepipedus (Piller \& Mitterpacher, 1783)

Souhairoux, Chemin de Doues (Henry, 1915: sub Abax striola); Pravillair (Valpelline) (Henry, 1915: sub Abax audoini); Comba di Valfreyde (Henry, 1935: sub Abax ater); Chez Collet (Ollomont) m 1280 (LB); Glacier (Ollomont) m 1500-1600 (LB); Thoules m 1050, De Bernardi leg. (Focarile, 1975); Prelé (Oyace) m 1100 (LB).

Le popolazioni valdostane di A. parallelepipedus sono di probabile origine transalpina. Alla luce delle attuali conoscenze, questa entità pare occupare solo la parte centrale della Valle d'Aosta: presente nella Valle del Gran San Bernardo e nella Valpelline, essa si spinge verso Sud sino alla bassa Valle di Cogne (che è anche il limite meridionale conosciuto in Valle D'Aosta) e al Vallone di Pila, e verso Est sino a Saint Marcel (cfr. Cerutti, 1932: sub Abax striola; Focarile, 1975: sub Abax ater; Henry, 1915: sub Abax audoini e sub Abax striola, 1935: sub Abax ater; Iehl, 1909: sub Abax striola), mentre sembra assente nelle valli valdostane della testata e in quelle più orientali (cfr. Allegro \& Chiarabaglio, 2008; Allegro et al., 2011; Bisio, 2006, 2013b; Bisio et al., 2012, in stampa; Bisio \& Giuntelli, 2014). La particolare distribuzione della specie fa pensare a una penetrazione dal versante svizzero attraverso il Colle del Gran San Bernardo e forse, considerata la frequenza della specie in Valpelline, attraverso l'ampio Col Fenêtre alla testata del Vallone di Ollomont.

Corotipo: Europeo (EUR).

\section{Amara (Amara) aenea (De Geer, 1774)}

Rhins (Roisan) m 900 (LB); Chez Collet (Ollomont) m 1280 (LB); Ollomont-Col de Fenêtre (Born, 1900b); «De Valpelline à Ollomont» (Pavesi, 1904); Thoules m 1050 (LB); Oyace m 1350-1450 (LB); Bionaz m 1700 (LB). Corotipo: Paleartico (PAL).

68. Amara (Amara) convexior Stephens, 1828

Doues m 1100 (LB); Valpelline m 960 (LB); Chez Collet (Ollomont) m 1280 (LB); Thoules m 1050 (LB); Oyace m 1350-1450 (LB).

Corotipo: Sibirico-Europeo (SIE).

69. Amara (Amara) curta Dejean, 1828

Chez Collet (Ollomont) m 1280 (LB); Glacier (Ollomont) m 1500-1600 (LB); Oyace m 1350-1450 (LB); Bionaz m 1700 (LB).

Corotipo: Sibirico-Europeo (SIE).

70. Amara (Amara) eurynota (Panzer, 1796)

Rhins (Roisan) m 900 (LB); «Valpelline: chemin d'Ollomont» (Henry, 1915: sub acuminata); Prelé (Oyace) m 1100 (LB). Corotipo: Sibirico-Europeo (SIE).

71. Amara (Amara) familiaris (Duftschmid, 1812) Viou (Roisan) (Henry, 1935).

Corotipo: Sibirico-Europeo (SIE).

72. Amara (Amara) lunicollis Schiödte, 1837

Glacier (Ollomont) m 1500-1600 (LB); Oyace m 1350-1450 
(LB); Comba di Valfreyde (Valpelline) (Henry, 1935).

Corotipo: Oloartico (OLA).

73. Amara (Amara) nitida Sturm, 1825

Glacier (Ollomont) m 1500-1600 (LB); Thoules m 1050

(LB); Comba d'Arpisson (Valpelline) (Henry, 1938).

Corotipo: Asiatico-Europeo (ASE).

74. Amara (Amara) ovata (Fabricius, 1792)

Valpelline (Henry, 1915: sub trivialis).

Corotipo: Asiatico-Europeo (ASE).

75. Amara (Amara) similata (Gyllenhal, 1810)

Valpelline (Henry, 1935); Chez Collet (Ollomont) m 1280

(LB); Prelé (Oyace) m 1100 (LB).

Corotipo: Asiatico-Europeo (ASE).

76. Amara (Celia) bifrons (Gyllenhal, 1810)

Valpelline (Bischoff-Ehinger \& Stierlin, 1870: sub rufocincta; Pavesi, 1904: sub livida); Bionaz m 1700 (LB).

Corotipo: Centroasiatico-Europeo (CAE).

77. Amara (Celia) erratica (Duftschmid, 1812)

Valpelline (Bischoff-Ehinger \& Stierlin, 1870; Pavesi, 1904); «Valpelline: colline de Souhairoux» (Henry, 1915); Mont Durand (Conca di By, Ollomont) (Henry, 1938); Bivacco Savoie (Conca di By, Ollomont) m 2700 (LB); Lacs de Thoules (Conca di By, Ollomont) m 2250-2350 (LB); Laghi del Morion (Glacier, Ollomont) m 2550 (LB); Comba della Sassa (Bionaz) m 2500-2600 (LB); Lac Long (Lago di Placemoulin, Bionaz) m 2720 (LB); Alpage Bella Tza (Prarayer, Bionaz) m 2747 (LB); Comba d'Arpisson (Valpelline) (Henry, 1935).

Corotipo: Oloartico (OLA).

78. Amara (Celia) praetermissa (Gyllenhal, 1810) «Du Col de Fenêtre à Aoste» (Gaud, 1902: sub rufocincta); Conca di By (Ollomont) m 2000 (Henry, 1935, 1938); Lacs de Thoules (Conca di By, Ollomont) m 2250-2350 (LB); Lac Long (Lago di Placemolin, Bionaz) m 2600 (LB); Prarayer (Bionaz) (Henry, 1935); Comba d'Arpisson (Valpelline) (Henry, 1935).

Corotipo: Sibirico-Europeo (SIE).

79. Amara (Xenocelia) ingenua (Duftschmid, 1812) Thoules m 1050 (LB); Prelé (Oyace) m 1100 (LB); Oyace m 1350-1450 (LB); Bionaz m 1700 (LB).

Specie xerotermofila nota nelle Alpi Occidentali piemontesi di un'unica stazione (Bisio \& Giuntelli, 2008), A. ingenua pare invece relativamente più frequente in Valle d'Aosta, probabilmente favorita dalla marcata xericità; oltre che nella valle in oggetto, essa è stata osservata in Valtournenche
(Bisio et al., in stampa), in Val d'Ayas (Bisio, 2005, 2006) e nella Valle di Cogne (Allegro \& Viterbi, 2009).

Corotipo: Asiatico-Europeo (ASE).

80. Amara (Xenocelia) municipalis (Duftschmid, 1812)

Rhins (Roisan) m 900 (LB); Chez Collet (Ollomont) m 1280 (LB); Thoules m 1050 (LB); Prelé (Oyace) m 1100 (LB); Oyace m 1350 (LB); Bionaz m 1700 (LB).

Corotipo: Sibirico-Europeo (SIE).

81. Amara (Paracelia) quenseli quenseli (Schönherr, 1806)

Valpelline (Bischoff-Ehinger \& Stierlin, 1870: sub monticola; Pavesi, 1904; Magistretti, 1965); Ollomont-Col de Fenêtre (Born, 1900b); «Du Col de Fenêtre à Aoste» (Gaud, 1902); «Vallée d'Ollomont» (Pavesi, 1904); Lacs de Thoules (Conca di By, Ollomont) m 2250-2350 (LB); Laghi del Morion (Glacier, Ollomont) m 2550 (LB); Bivacco Spataro (Plan de la Sabbla, Bionaz) m 2800 (LB); Comba della Sassa (Bionaz) m 2500-2600 (LB); Ghiacciaio della Sassa (Bionaz) m 3000 (LB); Lac Long (Lago di Placemoulin, Bionaz) m 2720 (LB); Prarayer (Henry, 1935, 1938); Rifugio Collon (Comba d'Oren, Lago di Placemoulin, Bionaz) m 2500 (LB); Bivacco Pra de Dieu (Comba d'Arbiére, Bionaz) m 2530 (LB); Colle di Saint-Barthélemy-Oyace (Born, 1900b); Comba d'Arpisson (Henry, 1935, 1938).

COROTIPO: Oloartico (OLA).

82. Amara (Percosia) equestris equestris (Duftschmid, 1812)

Ollomont-Col de Fenêtre (Born, 1900b); «De Valpelline à Ollomont» (Pavesi, 1904); «Valpelline: chemin d'Ollomont» (Henry, 1915); Les Crêtes (Ollomont) (Henry, 1938); Glacier (Ollomont) m 1500-1600 (LB); Conca di By (Glacier, Ollomont) m 2100 (LB); Comba di Breuson (Valpelline) (Henry, 1935); Bionaz m 1700 (LB); La Ferrere (Bionaz) m 1650 (LB).

Alcune tra le vecchie segnalazioni sopra citate potrebbero riferirsi alla specie seguente.

Corotipo: Asiatico-Europeo (ASE).

83. Amara (Percosia) infuscata (Putzeys, 1866) Col Champillon (Ollomont) m 2400-2500 (Bisio, 2009b). Corotipo: Sibirico-Europeo (SIE).

84. Amara (Bradytus) apricaria (Paykull, 1790) La Cleyva (Valpelline) (Henry, 1915); Les Crêtes (Ollomont) (Henry, 1938); Rey (Ollomont) (Henry, 1915); Comba di Breuson (Valpelline) (Henry, 1935); Bionaz m 1700 (LB).

Corotipo: Oloartico (OLA). 
85. Amara (Bradytus) consularis (Duftschmid, 1812) «Gignod: chemin de Valpelline» (Henry, 1915); Valpelline (Henry, 1915, 1916); Bionaz m 1700 (LB).

Corotipo: Asiatico-Europeo (ASE).

86. Amara (Curtonotus) aulica (Panzer, 1796)

Valpelline (Pavesi, 1904: sub spinipes; Henry, 1938; Magistretti, 1965); Glacier (Ollomont) m 1500-1600 (LB); Oyace m 1350-1450 (LB); Conca di By (Ollomont) (BischoffEhinger \& Stierlin, 1870: sub picea; Colle di Saint-Barthélemy-Oyace (Born, 1900b); Comba di Valfreyde (Valpelline) m 1500 (Henry, 1916); Viou (Roisan) (Henry, 1935).

COROTIPO: Oloartico (OLA).

87. Panagaeus (Panagaeus) bipustulatus (Fabricius, 1775)

«Valpelline: chemin d'Ollomont» (Henry, 1915).

P. bipustulatus è una specie che popola terreni asciutti e sabbiosi (Magistretti, 1965). Nelle Alpi Occidentali è nota solo di alcune stazioni valdostane (Henry, 1915; Magistretti, 1965; Bisio et al., 2012, in stampa; Casale com. pers., 2014). COROTIPO: Europeo (EUR).

88. Chlaeniellus nitidulus (Schrank, 1781)

«Doues: sentier des Leygé» (Henry, 1915).

C. nitidulus è noto di diverse stazioni valdostane. La segnalazione di Henry (1915) è quindi verosimile, anche se, data la predilezione della specie per greti sabbiosi e limosi, è probabile che si tratti di una popolazione molto localizzata come nel caso di altre entità dalle abitudini simili (Bembidion genei $\mathrm{e}$ Acupalpus flavicollis) censite in Valpelline. Corotipo: Centroasiatico-Europeo (CAE).

89. Licinus (Licinus) depressus (Paykull, 1790) Thoules m 1050, 1 es. 2.IV.2014 (LB);

Elemento dalle spiccate esigenze xerotermofile, $L$. depressus è probabilmente penetrato in Valle d'Aosta nel corso di una fase climatica xerica e/o ipsotermica (interglaciale e/o post-Glaciale) e vi è rimasto poi fino all'epoca attuale favorito dal clima, secondo l'ipotesi già avanzata da Focarile (1974) per altre entità dal praeferendum igrotermico simile. Secondo Casale (1977), il tipo di popolamento è incerto: potrebbe infatti trattarsi sia di una specie di origine transalpina, sia di un elemento di origine alpina sud-occidentale. Segnalata per la prima volta in Valle d'Aosta da Casale (1977) che ne cita diverse stazioni (quasi tutte dei dintorni di Aosta), la specie è stata recentemente trovata, oltre che nella stazione citata, in Valtournenche (Bisio et al., in stampa) e nella Valle di Cogne (Bisio, dati inediti).

Corotipo: Asiatico-Europeo (ASE).

90. Licinus (Licinus) cassideus (Fabricius, 1792) Frissonia di Sotto (Valpelline) m 1100, 1 es. 7.IV.2014 (LB).
Specie xerotermofila presente in buona parte dell'Italia peninsulare (cfr. Magistretti, 1965; Focarile, 1974), L. cassideus è stato segnalato per la prima volta in Valle d'Aosta da Focarile (1974). La specie è nota anche della Valle di Cogne (Bisio \& Giuntelli, dati inediti).

Corotipo: Centroeuropeo (CEU).

91. Badister (Badister) bullatus (Schrank, 1798)

Glacier (Ollomont) m 1500-1600 (LB); Thoules m 1050 (LB); Bionaz m 1700 (LB); La Ferrere (Bionaz) m 1650 (LB). COROTIPO: Oloartico (OLA).

92. Anisodactylus (Anisodactylus) binotatus (Fabricius, 1787)

Rhins (Roisan) m 900 (LB); Valpelline (Henry, 1935); Thoules m 1050 (LB); Oyace m 1350-1450 (LB); Prelé (Oyace) m 1100 (LB).

COROTIPO: Asiatico-Europeo (ASE).

93. Bradycellus (Bradycellus) caucasicus (Chaudoir, 1846)

Doues m 1000, 1 es. 10.IV.2013 (LB); Thoules m 1050, 1 es. 10.IV.2013 (LB).

CoRotipo: Sibirico-Europeo (SIE).

94. Acupalpus (Acupalpus) flavicollis (Sturm, 1825) Doues m 1100 (LB).

COROTIPO: Europeo (EUR).

95. Ophonus (Metophonus) laticollis Mannerheim, 1825

Glacier (Ollomont) m 1500-1600 (LB); Bionaz m 1700

(LB); La Ferrere (Bionaz) m 1650 (LB).

COROTIPO: Sibirico-Europeo (SIE).

96. Ophonus (Metophonus) rufibarbis (Fabricius, 1792)

Valpelline (Henry, 1935); Glacier (Ollomont) m 1500-1600 (LB); Thoules (Sciaky, 1987); ibidem a m 1050 (LB); Oyace m 1350-1450 (LB); Bionaz m 1700 (LB).

CoRotipo: Turanico-Europeo-Mediterraneo (TEM).

97. Cryptophonus tenebrosus (Dejean, 1829)

Frissonia di Sotto (Valpelline) m 1100 (LB); Chez Collet (Ollomont) m 1280 (LB); Prelé (Oyace) m 1100 (LB).

Corotipo: W-Paleartico (WPA).

98. Pseudoophonus (Pseudoophonus) griseus (Panzer, 1796)

Rhins (Roisan) m 900 (LB); Comba di Verdzignola (Valpelline) (Henry, 1938: sub Ophonus).

COROTIPO: Paleartico (PAL). 
99 Pseudoophonus (Pseudoophonus) rufipes (De Geer, 1774)

Rhins (Roisan) m 900 (LB); Valpelline (Henry, 1915: sub pubescens, 1935: sub pubescens); ibidem a m 960 (LB); Chez Collet (Ollomont) m 1280 (LB); Rey (Ollomont) (Henry, 1915: sub pubescens); Glacier (Ollomont) m 1500-1600 (LB); Thoules m 1050 (LB); Prelé (Oyace) m 1100 (LB); Oyace m 1350-1450 (LB); Bionaz (Henry, 1935: sub pubescens); ibidem a m 1700 (LB); La Ferrere (Bionaz) m 1650 (LB).

COROTIPO: Paleartico (PAL).

100. Harpalus (Harpalus) affinis (Schrank, 1781) Rhins (Roisan) m 900 (LB); «Du Col de Fenêtre à Aoste» (Gaud, 1902: sub aeneus); Valpelline (Henry, 1915: sub aeneus); Chez Collet (Ollomont) m 1280 (LB); Les Crêtes (Ollomont) (Henry, 1938: sub aeneus); Conca di By (Ollomont) (Henry, 1935: sub aeneus); Comba di Breuson (Valpelline) (Henry, 1935: sub aeneus); Thoules m 1050 (LB); Oyace m 1350 (LB); Prelé (Oyace) m 1100 (LB); Bionaz (Henry, 1935: sub aeneus); ibidem a m 1700 (LB); La Ferrere (Bionaz) m 1650 (LB); Comba di Verdzignola (Valpelline) (Henry, 1938: sub aeneus); Comba d'Arpisson (Valpelline) (Henry, 1938: sub aeneus).

Corotipo: Asiatico-Europeo (ASE).

101. Harpalus (Harpalus) distinguendus (Duftschmid, 1812)

Rhins (Roisan) m 900 (LB); Valpelline (Henry, 1915); Rey (Ollomont) (Henry, 1915); Thoules m 1050 (LB); Oyace m 1350 (LB).

Corotipo: Paleartico (PAL).

102. Harpalus (Harpalus) rubripes (Duftschmid, 1812) Doues m 1000 (LB); Valpelline (Henry, 1915; 1938); Ollomont-Col de Fenêtre (Born, 1900b: sub sobrinus); «De Valpelline à Ollomont» (Pavesi, 1904: sub rubripes var. sobrinus); Rey (Ollomont) (Henry, 1915); Glacier (Ollomont) m 1500-1600 (LB); Conca di By (Ollomont) m 2000 (Henry, 1915, 1938); Thoules m 1050 (LB); Oyace m 13501450 (LB); Bionaz (Henry, 1938); La Ferrere (Bionaz) m 1650 (LB); Prarayer (Bionaz) m 2000 (Henry, 1915); Comba d'Arpisson (Valpelline) (Henry, 1935).

COROTIPO: Asiatico-Europeo (ASE).

103. Harpalus (Harpalus) laevipes Zetterstedt, 1828 Chez Collet (Ollomont) m 1280 (LB); Champillon (Ollomont) (Henry, 1938: quadripunctatus); Conca di By (Ollomont) (Henry, 1938: quadripunctatus).

Corotipo: Asiatico-Europeo (ASE).

104. Harpalus (Harpalus) atratus Latreille, 1804 Valpelline (Henry, 1915); Prelé (Oyace) m 1100 (LB); Comba di Verdzignola (Valpelline) (Henry, 1938).

COROTIPO: Europeo (EUR).
105. Harpalus (Harpalus) luteicornis (Duftschmid, 1812)

Comba de Livournea (Bionaz) m 2100 (Henry, 1915).

COROTIPO: Europeo (EUR).

106. Harpalus (Harpalus) solitaris Dejean, 1829

Valpelline (Pavesi, 1904: sub fuliginosus); Souhairoux (Valpelline) (Henry, 1915: sub chevieri); Ollomont-Col de Fenêtre (Born, 1900b: sub fuliginosus); Ollomont (Pavesi, 1904: sub fuliginosus); Col Champillon (Ollomont) m 2700 (LB); Lacs de Thoules (Conca di By, Ollomont) m 22502350 (LB); Alpage Bella Tza (Prarayer) m 2747 (LB); Colle di Saint-Barthélemy-Oyace (Born, 1900b: sub fuliginosus). COROTIPO: Oloartico (OLA).

107. Harpalus (Harpalus) latus (Linné, 1758)

Valpelline (Henry, 1935); Bionaz (Henry, 1938).

COROTIPO: Asiatico-Europeo (ASE).

108. Harpalus (Harpalus) sulphuripes sulphuripes Germar, 1824

Comba di Verdzignola (Valpelline) (Henry, 1938).

COROTIPO: Europeo-Mediterraneo (EUM).

109. Harpalus (Harpalus) fuscicornis Ménétriés, 1832

Valpelline, 1 es. 23.VI.1971, Cosimi leg. (G. Franzini com. pers., 2013).

Specie non comune segnalata solo di recente in Francia (Ponel \& Coulon, 2013) e in Svizzera (Marggi et al., 2010) e segnalata dell'Italia Nord-orientale da Casale \& Vigna Taglianti (2005). In Valle d'Aosta H. fuscicornis è noto anche di Arpuilles (G. Franzini, com pers., 2013) e della Valle di Cogne (Götz com. pers., 2012).

COROTIPO: Paleartico (PAL).

110. Harpalus (Harpalus) honestus (Duftschmid, 1812)

Souhairoux (Valpelline) (Henry, 1915); Chez Collet (Ollomont) m 1280 (LB); Conca di By (Glacier, Ollomont) m 2000 (LB); Bionaz m 1700 (LB); Prarayer m 2000 (LB); Comba d'Arpisson (Valpelline) (Henry, 1935).

Corotipo: Sibirico-Europeo (SIE).

\section{Harpalus (Harpalus) rufipalpis rufipalpis Sturm, 1818}

Valpelline (Pavesi, 1904: sub rufitarsis); Valpelline m 960 (LB); Chez Collet (Ollomont) m 1280 (LB); Glacier (Ollomont) m 1500-1600 (LB); Conca di By (Glacier, Ollomont) m 2100 (LB); Mont Durand (Ollomont) (Henry, 1938: sub rufitarsis); Comba di Berrio (Ollomont) (Henry, 
1938: sub rufitarsis); Oyace m 1350-1450 (LB); Bionaz m 1700 (LB); Comba di Verdzignola (Valpelline) (Henry, 1938: sub rufitarsis).

Corotipo: Sibirico-Europeo (SIE).

112. Harpalus (Harpalus) serripes (Quensel in Schönherr, 1806)

Valpelline (Henry, 1935; Magistretti, 1965); ibidem a m 960 (LB).

CoRotiPo: Paleartico (PAL).

113. Harpalus (Harpalus) tardus (Panzer, 1797) Rhins (Roisan) m 900 (LB); «de Valpelline à Ollomont» (Pavesi, 1904); Ollomont-Col de Fenêtre (Born, 1900b); Doues m 1000 (LB); Chez Collet (Ollomont) m 1280 (LB); Les Crêtes (Ollomont) (Henry, 1938); Conca di By (Ollomont) (Henry, 1938); Thoules m 1050 (LB); Oyace m 13501450 (LB); Bionaz m 1700 (LB); Prarayer (Bionaz) (Henry, 1915); Comba d'Arpisson (Valpelline) (Henry, 1938); Viou (Roisan) (Henry, 1935).

Corotipo: Asiatico-Europeo (ASE).

114. Harpalus (Harpalus) anxius (Duftschmid, 1812) Thoules m 1050 (LB); Oyace m 1350-1450 (LB).

Corotipo: Paleartico (PAL).

115. Harpalus (Harpalus) pumilus Sturm, 1818

Souhairoux (Valpelline) (Henry, 1915: sub picipennis).

Corotipo: Paleartico (PAL).

116. Parophonus (Parophonus) maculicornis (Duftschmid, 1812)

Valpelline (Henry, 1938: sub Ophonus).

Corotipo: S-Europeo (SEU).

117. Trichotichnus (Trichotichnus) laevicollis (Duftschmid, 1812)

Valpelline (Pavesi, 1904: sub Harpalus); «Valpelline: chemin d'Ollomont» (Henry, 1915: sub Harpalus); Chez Collet (Ollomont) m 1280 (LB); Glacier (Ollomont) m 1500-1600 (LB); Thoules m 1050 (LB); Oyace m 1350-1450 (LB); Bionaz m 1700 (LB); Colle di Saint-Barthélemy-Oyace (Born, 1900b); Comba d'Arpisson (Valpelline) (Henry, 1935: sub Harpalus).

CoRotipo: Centroeuropeo (CEU).

\section{Calathus (Calathus) fuscipes graecus Dejean,} 1831

Rhins (Roisan) m 900 (LB); Valpelline (Henry, 1915, 1935); Doues m 1100 (LB); Les Crêtes (Ollomont) (Henry, 1938); Chez Collet (Ollomont) m 1280 (LB); Glacier (Ollomont) m 1500-1600 (LB); Conca di By (Ollomont) m 2000 (Henry,
1915); Thoules m 1050 (LB); Oyace m 1350-1450 (LB); Bionaz m 1700 (LB); La Ferrere (Bionaz) m 1650 (LB); Prarayer (Bionaz) m 2000 (Henry, 1915); Viou (Roisan) (Henry, 1935). CoRotiPo: Europeo-Mediterraneo (EUM).

119. Calathus (Neocalathus) melanocephalus (Linné, 1758)

Rhins (Roisan) m 900 (LB); Doues m 1100 (LB); «Valpelline: chemin d'Ollomont» (Henry, 1915); Les Grangettes (Valpelline) (Henry, 1915); Champillon (Ollomont) (Henry, 1935); Chez Collet (Ollomont) m 1280 (LB); Les Crêtes (Ollomont) (Henry, 1938); Rey (Ollomont) (Henry, 1915); Glacier (Ollomont) m 1500-1600 (LB); Conca di By (Ollomont) (Henry, 1915); Lacs de Thoules (Conca di By, Ollomont) $\mathrm{m}$ 2250-2350 (LB); «du Col de Fenêtre à Aoste» (Gaud, 1902: sub alpinus); «de Aoste au Col Fenêtre» (Pavesi, 1904); Lac Long (Lago di Placemoulin, Bionaz) $\mathrm{m}$ 2600 (LB); Thoules m 1050 (LB); Prelé (Oyace) m 1100 (LB); Oyace m 1350-1450 (LB); Bionaz m 1700 (LB); La Ferrere (Bionaz) m 1650 (LB); Comba della Sassa (Bionaz) m 2400 (LB); Prarayer (Bionaz) (Henry, 1915, 1935); Comba d'Arbière (Bionaz) (Henry, 1915); Comba di Verdzignola (Valpelline) (Henry, 1938); Comba d'Arpisson (Valpelline) (Henry, 1935).

Corotipo: Paleartico (PAL).

120. Calathus (Neocalathus) cinctus Motschulsky, 1850

Mont Durand (Ollomont) (Henry, 1938: sub mollis); Bionaz (Henry, 1938: sub mollis).

Corotipo: W-Paleartico (WPA).

121. Calathus (Neocalathus) micropterus (Duftschmid, 1812)

Comba della Sassa (Bionaz) m 2400 (LB); Prarayer (Henry, 1935); Comba d'Arpisson (Valpelline) (Henry, 1938); Comba di Valfreyde (Valpelline) (Henry, 1935).

Corotipo: Oloartico (OLA).

122. Calathus (Neocalathus) ambiguus (Paykull, 1790)

Les Crêtes (Ollomont) (Henry, 1938).

Specie xerotermofila legata a suoli sciolti soleggiati, C. ambiguus è stato segnalato da Focarile (1975) di diverse stazioni valdostane.

Corotipo: Asiatico-Europeo (ASE).

123. Calathus (Neocalathus) erratus (C.R. Sahlberg, 1827)

Rhins (Roisan) m 900 (LB); Doues m 1000 (LB); Valpelline (Henry, 1915, 1935); «de Valpelline à Ollomont» (Pavesi, 1904); Ollomont-Col de Fenêtre (Born, 1900b); Les Crêtes 
(Ollomont) (Henry, 1938); Conca di By (Ollomont) (Henry, 1935); Lacs de Thoules (Conca di By, Ollomont) m 22502350 (LB); Thoules m 1050 (LB); Prelé (Oyace) m 1100 (LB); Prarayer (Bionaz) (Henry, 1935).

Corotipo: Sibirico-Europeo (SIE).

124. Sphodrus leucophthalmus (Linné, 1758)

«Valpelline, dans l'église» (Henry, 1915).

Corotipo: W-Paleartico (WPA).

125. Agonum (Agonum) muelleri muelleri (Herbst, 1784)

Valpelline (Pavesi, 1904); Les Crêtes (Ollomont) (Henry, 1938); Glacier (Ollomont) m 1500-1600 (LB); Colle di Saint-Barthélemy-Oyace (Born, 1900b: sub Platynus); La Ferrere (Bionaz) m 1650 (LB); Comba di Verdzignola (Valpelline) (Henry, 1938); Les Grangettes (Valpelline) (Henry, 1915: sub parumpunctatum); Comba d'Arpisson (Valpelline) (Henry, 1935).

Corotipo: Sibirico-Europeo (SIE).

126. Agonum (Melanagonum) viduum (Panzer, 1796) Doues m 1100 (LB); La Ferrere (Bionaz) m 1650 (LB). Corotipo: Sibirico-Europeo (SIE).

127. Agonum (Punctagonum) sexpunctatum (Linné, 1758)

«Valpelline: chemin d'Ollomont» (Henry, 1915).

Corotipo: Sibirico-Europeo (SIE).

128. Anchomenus (Anchomenus) dorsalis (Pontoppidan, 1763)

Doues m 1100 (LB); Rey (Ollomont) (Henry, 1915); Thoules m 1050 (LB).

Corotipo: Paleartico (PAL).

129. Limodromus assimilis (Paykull, 1790)

Rhins (Roisan) m 900 (LB); Doues m 1100 (LB); Valpelline (Henry, 1935: sub Platynus); Chez Collet (Ollomont) m 1280 (LB); Glacier (Ollomont) m 1500-1600 (LB); Thoules m 1050 (LB).

Corotipo: Sibirico-Europeo (SIE).

130. Paranchus albipes (Fabricius, 1796)

Doues m 1000 (LB).

Corotipo: Europeo-Mediterraneo (EUM).

131. Cymindis (Cymindis) humeralis (Geoffroy in Fourcroy, 1785)

Valpelline (Pavesi, 1904); Champillon (Ollomont) (Henry, 1935); Conca di By (Ollomont) (Henry, 1935); Colle di Saint-Barthélemy-Oyace (Born, 1900b).

Corotipo: Europeo (EUR).
132. Cymindis (Cymindis) scapularis scapularis Schaum, 1857

Glacier (Ollomont) m 1500-1600 (LB).

Corotipo: S-Europeo (SEU).

133. Cymindis (Cymindis) coadunata coadunata Dejean, 1825

Conca di By (Ollomont) (Henry, 1938).

Corotipo: Centroeuropeo (CEU).

134. Cymindis (Tarulus) vaporariorum (Linné, 1758) Valpelline (Pavesi, 1904); Ollomont (Pavesi, 1904); Ollomont-Col de Fenêtre (Born, 1900b); Les Crêtes (Ollomont) (Henry, 1938); Col Champillon (Ollomont) m 2700 (LB); Conca di By (Ollomont) (Bischoff-Ehinger \& Stierlin, 1870); Bivacco Savoie (Conca di By, Ollomont) m 2700 (LB); Lacs de Thoules (Conca di By, Ollomont) m 2250-2350 (LB); Laghi del Morion (Glacier, Ollomont) m 2550 (LB); Lago Incliousa (Glacier, Ollomont) m 2400 (LB); Plan Meulet (Valpelline) (Henry, 1938); Bivacco Spataro (Plan de la Sabbla, Bionaz) m 2800 (LB); Comba della Sassa (Bionaz) m 25002600 (LB); Lac Long (Lago di Placemoulin, Bionaz) m 2720 (LB); Rifugio Collon (Comba d'Oren, Lago di Placemoulin, Bionaz) m 2700 (LB); Alpage Bella Tza (Prarayer, Bionaz) m 2747 (LB); Bivacco Pra de Dieu (Comba d'Arbiére, Bionaz) $m$ 2400-2530 (LB); Colle di Saint-Barthélemy-Oyace (Born, 1900b); Comba d'Arpisson (Valpelline) (Henry, 1935); Comba di Valfreyde (Valpelline) (Henry, 1935).

Corotipo: Sibirico-Europeo (SIE).

135. Syntomus truncatellus (Linné, 1761)

Oyace m 1350 (LB); Bionaz m 1700 (LB).

Corotipo: Sibirico-Europeo (SIE).

136. Paradromius (Manodromius) linearis (Olivier, 1795)

Chez Collet (Ollomont) m 1280 (LB); Thoules m 1050 (LB). Corotipo: Europeo-Mediterraneo (EUM).

137. Dromius (Dromius) agilis (Fabricius, 1787) Chez Collet (Ollomont) m 1280, pl. es. 9.XII.2011 (LB). Nella stazione sopra citata sono stati rinvenuti numerosi esemplari rispettivamente di $D$. agilis, di $D$. fenestratus e di $D$. quadrimaculatus (le tre specie di Dromius più comuni e diffuse nelle Alpi Occidentali: cfr. Bisio, 1996) rimuovendo le placche corticali di alcuni vecchi aceri montani e di diversi abeti rossi localizzati lungo un pendio in esposizione occidentale. Nonostante la notevole quantità di materiale raccolto, non è stata invece rilevata la presenza di Dromius meridionalis Dejean, 1825, specie già segnalata della Valle D'Aosta da Carret (1905), da Dodero (1927), da Magistretti (1965) e da Focarile (1975). Il mancato rinvenimento della specie in questo biotopo 
forestale è quasi certamente da collegare alla sua maggior scelta xerotermofila che porta probabilmente la specie a rifuggire gli ambienti ombrosi e freschi colonizzati da queste essenze.

138. Dromius (Dromius) fenestratus (Fabricius, 1794) Chez Collet (Ollomont) m 1280, pl. es. 9.XII.2011 (LB). Corotipo: Europeo (EUR).

\section{Dromius (Dromius) quadrimaculatus (Linné,} 1758)

Chez Collet (Ollomont) m 1280, pl. es. 9.XII.2011 (LB). D. quadrimaculatus è un'entità prevalentemente diffusa in pianura (dove probabilmente è molto più comune di quanto non appaia: a tale proposito cfr. Scheffler, 1997; Felix \& van Wielink, 2008, 2011) e nella fascia submontana che raramente si spinge al di sopra dei $1000 \mathrm{~m}$ (cfr. Bisio, 1996). La quota sopra indicata rappresenta, a tutt'oggi, il record altitudinale della specie. In Valle d'Aosta questa entità è pressoché sconosciuta quasi certamente per difetto di ricerche: a tutt'oggi, infatti, essa è nota solo della stazione sopra citata e dei dintorni di Aymavilles (Bisio, dati inediti). È tuttavia probabile una sua presenza diffusa lungo il fondovalle valdostano. Corotipo: Europeo (EUR).

140. Calodromius spilotus (Illiger, 1798)

Chez Collet (Ollomont) m 1280, 1 es. su acero montano, 9.XII.2011 (LB).

Lo scrivente ha condotto per molti anni indagini sulla carabidofauna arboricola in molte valli dell'arco alpino occidentale. Tali indagini sono state rivolte quasi sempre a essenze arboree (soprattutto aceri montani, ma anche conifere come abeti rossi e larici) la cui corteccia si sfalda spontaneamente in placche facilmente asportabili manualmente (cfr. Bisio, 1996). Con questo metodo è stato possibile rilevare in molte stazioni la notevole di consistenza delle popolazioni delle tre specie di Dromius precedenti. Per contro, una specie notoriamente arboricola come $C$. spilotus è risultata apparentemente molto rara, tanto che nelle Alpi Occidentali italiane, oltre che della località citata, essa è nota di un'unica stazione piemontese (Bisio, 1996, 2013a). In realtà, come dimostrano le ricerche di Scheffler (1997) e di Felix \& van Wielink (2008, 2011), è probabile che questa entità sia molto più comune di quanto non sembri. Sicuramente i risultati delle ricerche sono molto influenzati dal metodo adottato: Felix \& van Wielink (2008, 2011), infatti, hanno dimostrato la notevole efficacia, per il censimento di questa specie e di altri Dromiini, dell'osservazione notturna dei tronchi e dell'impiego di fasce di cartone ondulato avvolte attorno al tronco e alle branche (metodo già precedentemente adottato da Casale et al., 1996). Non è tuttavia da escludere che la maggior o minor consistenza della popolazione censita sia, almeno in parte, da collegare al praeferendum altitudinale di C. spilotus (lo scrivente ha quasi sempre operato a quote comprese tra 500 e $1800 \mathrm{~m}$, mentre sia
Scheffler, sia Felix \& van Wielink hanno raccolto a campione in zone poco al di sopra del livello del mare) o alla specie arborea investigata. A questo proposito, è da rilevare che Scheffler (1997) - che ha effettuato le sue ricerche su una rosa di dieci generi di essenze arboree praticando la rimozione manuale delle placche corticali su vecchi alberi adatti allo scopo («geeigneten Altbäumen») - ha rilevato la presenza di una quantità molto elevata di esemplari di C. spilotus solo su Populus, a fronte di quantità molto inferiori o addirittura di nessun esemplare su altre essenze.

141. Lebia cruxminor (Linné, 1758)

Souhairoux (Valpelline) (Henry, 1915).

Corotipo: Paleartico (PAL).

\section{PRINCIPALI CARABIDOCENOSI RILEVATE}

CARABIDOCENOSI SILVICOLE DELLE FASCE SUBMONTANA, MONTANA E ARBUSTIVA. Come già osservato in altre valli valdostane (cfr. Bisio, 2013b; Bisio \& Giuntelli, 2014; Bisio et al., in stampa e dati inediti), la marcata xericità che caratterizza la fascia submontana nel tratto di valle tra Roisan e Valpelline condiziona la composizione delle carabidocenosi che popolano gli ambienti boschivi. Nelle aree sottoposte a campionamento mediante l'ausilio di trappole a caduta (pitfall traps) l'unico Carabide censito è stato Carabus intricatus, taxon che si conferma, una volta di più, come un elemento silvicolo ad ampia valenza ecologica, osservabile con una certa regolarità anche nelle formazioni forestali più xeriche come quelle a roverella. A questa specie, nei boschi igrofili che colonizzano le rive del Torrente Buthier, si aggiungono $\mathrm{Li}$ modromus assimilis (assai comune) e Notiophilus palustris (molto meno frequente). Come nella Valle di Saint-Barthélemy (cfr. Bisio, 2013b), anche in Valpelline pare assente Abax (Abax) continuus Ganglbauer, 1891, entità che in Valle d'Aosta sembra limitare la sua presenza alle valli più orientali (Bisio, 2006; Allegro \& Chiarabaglio, 2008; Bisio et al., 2012, in stampa).

La fascia montana è invece popolata dalla tipica coleotterocenosi silvicola a Pterosticbus multipunctatus la cui composizione è stata rilevata da Focarile (1974) in dieci stazioni valdostane. Formata, per quanto riguarda i Carabidi, dal consueto gruppo di specie (oltre a quella citata, Carabus nemoralis, C. depressus, C. intricatus, Nothiophilus biguttatus, Pterostichus oblongopunctatus, Trichotichnus laevicollis, Limodromus assimilis e Calathus micropterus) che caratterizza buona parte dei biotopi forestali valdostani, in Valpelline tale cenosi è arricchita da popolazioni diffuse di Abax parallelepipedus, entità di probabile 
origine transalpina che, allo stato delle attuali conoscenze, in Valle d'Aosta sembra occupare solo le valli più centrali (cfr. Focarile, 1975). Completano l'elenco delle specie silvicole note della fascia montana Leistus ferrugineus e L. spinibarbis, entità che paiono decisamente più rare, la cui presenza in Valpelline è testimoniata solo dalle vecchie segnalazioni di Henry (1915).

Con l'aumentare della quota il numero di elementi silvicoli rilevati diminuisce e nella fascia arbustiva ad Alnus viridis si riduce a un nucleo ristretto costituito solo da Pterostichus multipunctatus, Trichotichnus laevicollis e Calathus micropterus.

CARABIdocenosi delle Formazioni APERTE NELLE FASCE FORESTALI. Rispetto agli ambienti forestali, i prati falciabili del fondovalle sono popolati da carabidocenosi più numerose, anche se in alcune zone sono state censite solo specie nel complesso piuttosto comuni. 亡̀ il caso soprattutto della fascia submontana, dove le ricerche effettuate hanno consentito di rilevare solo la presenza di Poecilus cupreus, P. lepidus, Amara aenea, A. convexior, A. eurynota, A. municipalis, Anisodactylus binotatus, Pseudoophonus rufipes, Harpalus distinguendus, H. affinis, H. tardus, H. anxius, Calathus fuscipes, C. erratus e C. melanocephalus.

Nell'orizzonte montano il contingente di specie che popola le formazioni aperte tende a differenziarsi e ad arricchirsi con la comparsa di elementi maggiormente legati a tale fascia. Di notevole interesse è il rinvenimento in tale orizzonte di Licinus depressus e di $L$. cassideus, entità xerotermofile di rilevante pregio faunistico la cui presenza attesta, di fatto, l'accentuata xericità di alcuni ambienti del fondovalle già evidenziata da Focarile (1974: cartina Aree xerotermiche in Valle d'Aosta). Tra gli altri taxa censiti sono piuttosto numerosi gli Harpalini (Anisodactylus binotatus, Bradycellus caucasicus, Ophonus rufibarbis, O. laticollis, Cryptophonus tenebrosus, Pseudoophonus rufipes, P. griseus, Harpalus affinis, $H$. distinguendus, $H$. rubripes, H. laevipes, H. atratus, H. luteicornis, H. latus, H. sulphuripes, H. fuscicornis, $H$. honestus, $H$. rufipalpis, $H$. tardus, H. anxius, H. pumilus e Parophonus maculicornis) e le specie del genere Amara (A. aenea, A. convexior, A. curta, A. eurynota, A. familiaris, A. lunicollis, A. nitida, A. ovata, A. similata, A. bifrons, $A$. ingenua, A. municipalis, $A$. equestris, $A$. apricaria, $A$. consularis e $A$. aulica). È stata inoltre rilevata la presenza di diversi Pterostichini (Stomis pumicatus, Poecilus versicolor, $P$. cupreus, P. lepidus, Pterostichus strenuus, P. niger, $P$. melanarius e P. rhaeticus) e di alcuni Calathus (C. fuscipes, C. melanocephalus, C. cinctus, C. ambiguus e C. erratus). Completano infine l'elenco delle specie praticole segnalate Carabus problematicus, C. germarii, Trechus quadristriatus, Panagaeus bipustulatus, Badister bullatus, Agonum sexpunctatum, A. muelleri, Anchomenus dorsalis, Cymindis scapularis, Syntomus truncatellus, Paradromius linearis e Lebia cruxminor.

CARABIDOCENOSI DEGLI ORIZZONTI ALPINO, ALTO-ALPINO E NIVALE. Come descritto da Focarile (1987), gli orizzonti alpino e alto-alpino e nivale sono occupati da diverse carabidocenosi la cui fenologia è influenzata dal variare dell'umidità al suolo nel corso dell'estate. Alle specie la cui comparsa è legata all'elevato tasso di umidità del primo fondere delle nevi si sostituiscono gradualmente le entità via via meno esigenti più tardive che compaiono quando i nevai sono ormai scomparsi. $\grave{E}$ da rilevare che in alcune valli valdostane entrano spesso a far parte di queste carabidocenosi taxa ad areale ristretto e, pertanto, la loro composizione varia talora da valle a valle. È il caso della valle in oggetto, dove il contingente di specie orofile presenti ricalca quasi perfettamente quello osservato nella vicina Valle di Saint-Barthélemy (cfr. Bisio, 2013b), ma si differenzia in parte rispetto alle altre due valli confinanti, la Valtournenche e Valle del Gran San Bernardo.

Com'è noto, l'inizio estate è il momento della comparsa degli elementi perinivali in senso stretto (caratterizzanti il Nebrietum nivale sensu Focarile, 1973) che occupano durante le ore diurne i suoli saturi di acqua di fusione ai margini dei nevai e si spostano notte-tempo sulla superficie degli stessi per nutrirsi della microfauna portata in quota dalle correnti ascensionali (cfr. Focarile, 1987). Come nella Valle di SaintBarthélemy (cfr. Bisio, 2013b), delle tre specie presenti in territorio valdostano in Valpelline è stata rinvenuta solo Oreonebria castanea. È infatti da ritenere ragionevolmente certa l'assenza di Nebria ( $\mathrm{Ne}$ briola) cordicollis cordicollis Chaudoir, 1837, taxon che lungo il versante valdostano sinistro raggiunge il limite occidentale del suo areale in Valtournenche (cfr. Focarile, 1976, Bisio, 1999, 2006; Bisio et al., in stampa). Pare inoltre assente Oreonebria (Oreonebria) angusticollis (Bonelli, 1810), entità il cui areale a Est del Colle del Gran San Bernardo sembra far registrare un'ampia soluzione di continuità che abbraccia sia la valle in oggetto, sia la Valle di Saint-Barthélemy; la specie, infatti, ricompare solo in Valtournenche e in 
Val d'Ayas, dove è presente con popolazioni puntiformi (cfr. Focarile \& Casale, 1978; Bisio, 2006, 2007, 2013b; Bisio et al., in stampa).

A Oreonebria castanea si accompagna il corteggio di specie igrofile e frigofile a fenologia precoce e contratta che si affollano attorno ai nevai attratte dall'elevato tasso di umidità: Oreonebria picea, Carabus depressus, Amara erratica e le specie di Bembidion costituenti il Testedioletum sensu Focarile, 1973 (B. pyrenaeum, B. magellense, B. glaciale e B. bipunctatum). Con la graduale fusione del manto nevoso, $i$ taxa sin qui citati tendono a rarefarsi e sono sostituiti da Carabus concolor e Pterostichus multipunctatus entità relativamente meno esigenti in fatto di umidità, caratterizzate di conseguenza da una fenologia più prolungata. Quest'ultima specie, che è un elemento tendenzialmente silvicolo, nella valle in oggetto raggiunge quote intorno ai 2700-2800 m; si conferma ulteriormente, quindi, la sua tendenza a colonizzare regolarmente la prateria alpina, tendenza probabilmente favorita dall'assenza in valle di possibili barriere competitive rappresentate da altri Pterostichus orofili (cfr. Focarile, 1974, 1987). In Valpelline, infatti, sono assenti $P$. (Oreophilus) cribratus (Dejean, 1828) e P. (Oreophilus) spinolae (Dejean, 1828), specie che raggiungono il limite occidentale del loro areale lungo il versante sinistro della Valtournenche (cfr. Bisio et al., in stampa).

Con l'avanzare dell'estate, dopo la scomparsa degli ultimi nevai, compare il tipico contingente di entità xerofile a fenologia tardiva e prolungata (AmaretoCyminditetum sensu Focarile, 1973). Si tratta in buona parte di entità granivore legate a suoli più evoluti colonizzati dal pascolo alpino. Nella valle in oggetto il rappresentante più comune e diffuso di tale contingente è Cymindis vaporariorum; in sintopia con questa specie si osservano, in alternanza a seconda della stazione, C. humeralis, C. coadunata, Harpalus solitaris, H. honestus, Calathus melanocephalus, Amara quenseli, A. equestris, A. infuscata e A. pratermissa.

CARABIDOCENOSI RIPICOLE. La composizione delle carabidocenosi ripicole che popolano il reticolo idrografico della Valpelline (negli affioramenti idrici in quota, sulle rive dei torrenti che discendono lungo i fianchi della valle e nel greto del Torrente Buthier sino alla confluenza di quest'ultimo con il Torrente Artanavaz) è molto variegata in funzione del graduale riscaldamento delle acque al diminuire della quota e delle variazioni delle caratteristiche degli alvei (quantità e dimensioni dei sedimenti).
Alle quote più elevate la specie caratterizzante il Nebrietum fontinale (sensu Focarile, 1973) è Nebria laticollis, elemento frigofilo stenotermo legato ad acque fredde (cfr. Focarile \& Casale, 1978; Focarile, 1987; Bisio, 1999). Questa entità si osserva regolarmente nei biotopi sorgivi di diversi valloni (di solito come unico Carabide) e, talora, nei torrentelli convoglianti acque sorgive e di fusione dove è presente in sintopia con $N$. jockischii. Inoltre, una popolazione piuttosto consistente di $N$. laticollis è stata censita anche lungo le rive del Lago Fenêtre (bacino alimentato da acque di fusione e di ablazione situato poco a valle del colle omonimo) dove la specie è sintopica con elementi perinivali ( $\mathrm{Bem}$ bidion bipunctatum, B. magellense e Oreonebria castanea) trasgressivi dai nevai circostanti.

Più a valle, ai bordi dei torrenti a forte pendenza che discendono dai valloni e confluiscono nel Buthier, N. laticollis è sostituita da N. jockischii e $N$. rufescens, taxa meno esigenti in fatto di temperatura. Compare anche un primo gruppo, ancora numericamente ridotto, di Bembidion costituito da popolazioni molto abbondanti rispettivamente di $B$. incognitum, $B$. geniculatum e $B$. tibiale.

Gli alvei del Buthier e del Torrente Ollomont, suo principale affluente, sono caratterizzati da un greto costituito da detriti mediamente grossolani (soprattutto pietrisco e ghiaia), granulometria che permane sino all'imbocco della valle e che influenza in modo marcato la composizione della carabidofauna ripicola. Nebria jockischii e $N$. rufescens, ancora presenti nella parte alta della valle principale e nel Vallone di Ollomont, verso la bassa valle sono sostituite da N. picicornis. La comunità dei Bembidiini si arricchisce notevolmente di entità in gran parte legate ai greti sassosi. Alcune di queste risultano diffuse lungo le due aste fluviali (Bembidion varicolor, $B$. complanatus, $B$. geniculatum, $B$. tibiale, $B$. decorum, B. tetracolum, B. bualei e Sinechostictus ruficornis), altre, più legate a greti sabbiosi sembrano presenti con popolazioni molto più localizzate (Asaphidion caraboides, A. flavipes, Bembidion pygmaeum, B. punctulatum, B. azurescens, B. ascendens, B. fasciolatum, B. femoratum e Sinechostictus decoratus). Alla luce degli esiti delle ricerche condotte da Ravizza (1972) e di quelle più recenti effettuate dallo scrivente, paiono invece molto meno comuni Ocydromus longipes, Sinechostictus doderoi, S. elongatus e Chlaeniellus nitidulus. Infatti, le prime due specie sono state censite da Ravizza (1972) in numero molto ridotto di esemplari, mentre la presenza in Valpelline di S. elongatus e C. ni- 
tidulus è testimoniata solo dalle vecchie segnalazioni di Henry (1916) mai più confermate. Altrettanto rara pare anche Loricera pilicornis, entità della quale è stato trovato un unico esemplare nel Vallone di Ollomont. A causa delle caratteristiche granulometriche della maggioranza dei corsi d'acqua, la presenza in valle di elementi luticoli risulta invece molto limitata: sono state censite solo poche specie (Bembidion quadrimaculatum, B. genei, Acupalpus flavicollis e Agonum viduum), localizzate lungo le rive di piccoli ruscelli fangosi.

\section{CONCLUSIONI}

Le 141 specie di Carabidae censite in Valpelline rappresentano il $47 \%$ delle 305 note che, secondo un elenco elaborato dalla lista fornita da Casale \& Vigna Taglianti (1993) e aggiornato sia con reperti inediti, sia con alcuni dati tratti dai lavori di Henry, sono sino a oggi note delle Alpi Pennine. Come già rilevato per la Valle di Saint-Barthélemy (cfr. Bisio, 2013b), anche in questo caso si tratta di una carabidofauna relativamente più povera come numero di taxa rispetto a quella che caratterizza il settore faunistico del Monte Rosa-Biellese (cfr. Bisio, 2006; Bisio et al., 2012, in stampa). Ciò è dovuto al fatto che i numerosi endemiti e stenoendemiti che popolano diffusamente le valli del Biellese $\mathrm{e}$, in parte, quelle più orientali del versante sinistro della Valle d'Aosta raggiungono quasi tutti il limite occidentale del proprio areale tra la Valle di Gressoney e la Valtournenche (cfr. Bisio, 2006). Il contingente di queste specie ad areale ristretto, già significativamente diminuito di numero in quest'ultima valle, nella Valle di Saint-Barthélemy e nella valle in oggetto si riduce a un unico rappresentante (Carabus concolor). Secondo quanto già discusso da Focarile (1974), il graduale aumento della xericità a mano a mano che ci si sposta verso la parte centrale della Valle d'Aosta - xericità che lungo la sinistra orografica è accentuata dall'esposizione meridionale - probabilmente ha costituito, nel corso del post-würmiano, una barriera alla penetrazione verso Ovest delle specie più igrofile e stenoterme proprie e spesso endemiche del settore Monte Rosa-Biellese, selezionandole in base al loro praeferendum igrotermico e alla loro bassa capacità di dispersione. Per contro, il minor tasso di precipitazioni che caratterizza la Valpelline può aver favorito l'insediamento nell'area in oggetto del nucleo di specie più xerofile e xerotermofile (v. Catalogo topografico) che la popolano e che invece sembrano meno frequenti nelle valli valdostane più orientali.
La Valpelline sembra inoltre essere stata più permeabile a popolamenti di origine transalpina, attraverso la cosiddetta "via dei colli" (sensu Focarile, 1974), da parte di alcune entità che, ampiamente diffuse sul versante svizzero e/o su quello francese, potrebbero essere penetrate in sede cisalpina, favorite dall'optimum termico post-würmiano (cfr. Casale \& Vigna Taglianti, 1993). Oltre che per le specie più comuni presenti in buona parte del territorio valdostano (Carabus nemoralis, Bembidion pyrenaeum e Pterostichus multipunctatus), si può ipotizzare quest'origine per le popolazioni presenti nella valle in oggetto di Loricera pilicornis, Abax parallelepipedus e Licinus depressus (con qualche dubbio per quest'ultima specie, data l'incertezza del tipo di popolamento che ne ha determinata la presenza in Valle d'Aosta: v. Catalogo topografico).

Anche sotto l'aspetto qualitativo, rispetto alle valli valdostane più orientali, emerge un'evidente banalizzazione delle carabidofauna con una netta prevalenza degli elementi a più vasto areale (Tab. $1 \mathrm{e}$ Fig. 7): ben 135 (pari al 96\% del totale dei taxa censiti) dei quali

Tab. 1. Distribuzione percentuale dei corotipi.

\begin{tabular}{lcc}
\hline Corotipi & $\mathrm{N}$. & $\%$ \\
\hline Oloartici & 13 & 9,22 \\
OLA & 15 & 10,64 \\
PAL & 4 & 2,84 \\
WPA & 19 & 13,48 \\
ASE & 24 & 17,02 \\
SIE & 1 & 0,71 \\
CEM & 3 & 2,13 \\
CAE & 2 & 1,42 \\
TEM & 0 & 0,00 \\
TUE & 0 & 0,00 \\
AFP & 6 & \\
\hline Europei & 6,26 \\
EUM & 25 & 17,73 \\
EUR & 17 & 12,06 \\
CEU & 6 & 4,26 \\
SEU & & \\
\hline Mediterranei & 0 & 0,00 \\
MED & & \\
\hline Endemici & & 2,13 \\
ALPW & 3 & 1,42 \\
ALPS & 2 & 0,71 \\
ALAP & 1 & 100,00 \\
\hline Totale & 141 &
\end{tabular}


81 (pari al 58\%) a gravitazione più settentrionale (oloartici sensu lato) e 54 (pari al 38\%) a corotipo europeo sensu lato. Nessuna specie a corotipo mediterraneo è stata censita in valle. Le entità endemiche (W-Alpine, S-Alpine e Alpino-Appenniniche) presenti in Valpelline risultano essere solo 6 (pari al 4\%); tre di queste (Oreo-

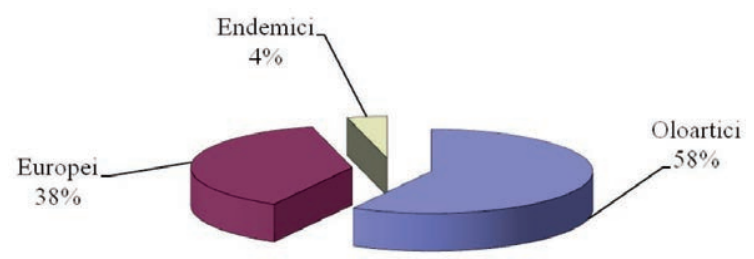

Fig. 7. Distribuzione percentuale dei corotipi. nebria picea, Bembidion magellense e P. multipunctatus) sono comunque taxa che presentano un areale relativamente ampio e due (Nebria laticollis e Bembidion pallidicorne) popolano quasi per intero l'arco alpino occidentale. Come già evidenziato, l'unica specie presente in valle ad avere un areale effettivamente più ristretto è Carabus concolor.

\section{RINGRAZIAMENTI}

Desidero ringraziare gli amici Achille Casale e Gianni Allegro per la lettura critica del testo e per i preziosi suggerimenti, Matteo Negro per la redazione della tavola concernente la valle in oggetto, Gabriele Franzini per la comunicazione di dati corologici e mia moglie Anna Maria che mi ha spesso accompagnato nelle numerose escursioni effettuate in Valpelline.

\section{BIBLIOGRAFIA}

Allegro G., Bisio L., 2007 - La carabidofauna della Riserva naturale del Mont Mars (Fontainemore, Aosta) (Coleoptera Carabidae). Revue Valdôtaine d'Histoire Naturelle, 60(2006): 73-92.

Allegro G., Bisio L., Negro M., 2011 - I Carabidi di Val Veny e Val Ferret (Coleoptera Carabidae) (Valle d'Aosta, Italia). Bollettino della Società Entomologica Italiana, 143(2): 59-76.

Allegro G., Chiarabaglio P., 2008 - I Carabidi del Parco naturale del Mont Avic (Valle d'Aosta) (Coleoptera, Carabidae). Revue Valdôtaine d'Histoire Naturelle, 61-62(2007-2008): 179-188.

Allegro G., Viterbi R., 2009 - Note faunistiche ed ecologiche sui carabidi del Parco Nazionale del Gran Paradiso (Coleoptera Carabidae). Rivista Piemontese di Storia Naturale, 30: 129-147.

Bischoff-Ehinger A., Stierlin G., 1870 - Reise in die italienischen Hochgebirge des Piemonts. Mitteilungen der Schweizerischen Entomolögischen Gesellschaft, 3(4): 159-175.

BARETti M., 1893 - Geologia della provincia di Torino. Casanova editore, 732 pp.

BISIO L., 1996 - Risultati di due anni di ricerche sui Dromiini corticicoli in alcune stazioni del Piemonte (Coleoptera Carabidae). Rivista Piemontese di Storia Naturale, 16(1995): 121-167.

Bisıo L., 1999 - Note sulle popolazioni di alcune Nebria del subg. Nebriola e di Nebria crenatostriata (Coleoptera Carabidae). Rivista Piemontese di Storia Naturale, 19(1998): 151-192.

Bisio L., 2002 - Terzo contributo alla conoscenza di Carabus del subg. Orinocarabus del Piemonte: Carabus concolor Fabricius, 1792 (Coleoptera Carabidae). Rivista Piemontese di Storia Naturale, 23: 155-166.

Bisio L., 2005 - Secondo contributo alla conoscenza del genere Amara in Piemonte e in Valle d'Aosta: osservazioni su alcune specie del subg. Celia e del subg. Bradytus (Coleoptera Carabidae). Rivista Piemontese di Storia Naturale, 26: 211-223.

Bisio L., 2006 - I Coleotteri Carabidi della Val d'Ayas (Valle d'Aosta) (Coleoptera Carabidae). Revue Valdôtaine d'Histoire Naturelle, 59(2005): 45-77.

Bisio L., 2007 - Contributo alla conoscenza di Oreonebria del Piemonte e della Valle d'Aosta: Oreonebria (Oreonebria) angusticollis (Bonelli, 1809) (Coleoptera Carabidae). Rivista Piemontese di Storia Naturale, 28: 165-180.

Bisio L., 2008 - Secondo contributo alla conoscenza di Oreonebria del Piemonte e della Valle d'Aosta: Oreonebria castanea e le specie del gruppo "picea"(Coleoptera Carabidae). Rivista Piemontese di Storia Naturale, 29: 177-209.

Bisio L., 2009a - Note corologiche e ecologiche su alcuni Bembidiini perinivali delle Alpi Occidentali italiane (Coleoptera Carabidae). Rivista Piemontese di Storia Naturale, 30: 95-129.

Bisio L., 2009b - Terzo contributo alla conoscenza del gen. Amara Bonelli, 1810 in Piemonte e in Valle d'Aosta: osservazioni sulle specie del subg. Percosia (Coleoptera Carabidae). Rivista Piemontese di Storia Naturale, 30: 149-161. 
BIsIo L., 2013a - I Coleotteri Carabidi della Val Chisone (Alpi Cozie) (Coleoptera Carabidae). Rivista Piemontese di Storia Naturale, 34: 181-238.

Bisıo L., 2013b - I Coleotteri Carabidi della Valle di Saint-Barthélemy (Valle d'Aosta) (Coleoptera Carabidae). Revue Valdôtaine d'Histoire Naturelle, 67: 89-116.

Bisio L., Giuntelli P., 2008 - I Coleotteri Carabidi della Val Varaita (Coleoptera Carabidae). Rivista Piemontese di Storia Naturale, 29: 225-278.

Bisio L., Giuntelli P., 2014 - I Coleotteri Carabidi della Valgrisenche (Valle d'Aosta) (Coleoptera Carabidae). Revue Valdôtaine d'Histoire Naturelle, 68: 25-53.

Bisio L., Negro M., Allegro G., 2012 - I Coleotteri Carabidi della Valle di Gressoney (Valle d'Aosta) (Coleoptera Carabidae). Revue Valdotaine d'Histoire Naturelle, 66: 5-43.

Bisio L., Negro M., Giuntelli P., in stampa - I Coleotteri Carabidi della Valtournenche (Valle d'Aosta) (Coleoptera Carabidae). Revue Valdôtaine d'Histoire Naturelle, 69.

BORN P., 1900a - Meine Excursion von 1899. Societas Entomologica, 15(2): 11-12.

BORN P., 1900b - Meine Excursion von 1899. Societas Entomologica, 15(3): 18-20.

Born P., 1904 - Die Carabenfauna des Aostatales. Societas Entomologica, 19(15): 113-114.

CARret A. 1905 - Escursioni e caccie entomologiche in qualche valle del Piemonte. VII. Da Aosta a Cogne per Aymaville. Rivista coleotterologica italiana, 3(3): 70-79.

Casale A., 1977 - Reperti di Licinus (s. str.) depressus (Paykull) in Valle d'Aosta. Sistematica del genere Licinus e delle specie italiane del "gruppo depressus" (Col. Carabidae). Revue Valdôtaine d'Histoire Naturelle, 31: 84-92.

Casale A., Giachino P.M., PAntaleoni R., 1996 - Life history and pre-imaginal stages of Dromius meridionalis (Coleoptera: Carabidae: Dromiini) in Sardinia. Acta Societatis Zoologicae Bohemoslavacae: 60: 363-371.

Casale A., Sturani M., Vigna Taglianti A., 1982 - Coleoptera Carabidae I. Introduzione, Paussinae, Carabinae. Fauna d'Italia, XVIII, Calderini, Bologna, 499 pp.

Casale A., Vigna Taglianti A., 1993 - I Coleotteri Carabidi delle Alpi occidentali e centro-occidentali (Coleoptera Carabidae). Biogeographia (Il popolamento delle Alpi occidentali), 16(1992): 331-399.

Casale A., Vigna Taglianti A., 2005 - Coleotteri Caraboidei delle Alpi e Prealpi centrali e orientali, e loro significato biogeografico (Coleoptera, Caraboidea). Biogeographia, 26: 129-201.

Casale A., Vigna Taglianti A., Brandmayr P., Colombetta G. 2006 - Insecta Coleoptera Carabidae (Carabini, Cychrini, Trechini, Abacetini, Stomini, Pterostichini). In: Ruffo S., Stoch F. (eds.), Ckmap (Checklist and distribution of the Italian fauna). Memorie del Museo Storia Naturale Verona, 2. serie, Sezione Scienze della vita, 17: 159-164, with data on CD-Rom.

Cerutti C., 1932 - Les insectes du Vallon du Grand-Saint-Bernard. Bulletin de la Société de la Flore Valdôtaine, 21: 32-39.

Dal Piaz G.V. (coord.), 1992a - Guide Geologiche Regionali, vol. 3, Le Alpi dal Monte Bianco al Lago Maggiore, Parte prima. Pubblicazione a cura della Società Geologica Italiana. Ed. Be. Ma., 310 pp.

Dal Piaz G.V. (coord.), 1992b - Guide Geologiche Regionali, vol. 3, Le Alpi dal Monte Bianco al Lago Maggiore, Parte seconda. Pubblicazione a cura della Società Geologica Italiana. Ed. Be. Ma., 202 pp.

Dodero A., 1927 - Coleotteri di Valsavaranche (Valle d'Aosta). Memorie della Società Entomologica Italiana, 6: 223-226.

FELIX R., VAN WiELINK P., 2008 - On the biology of Calodromius bifasciatus and related species in "De Kaaistoep" (Coleoptera: Carabidae). Entomologische Berichten, 68(6): 198-209.

FELIX R., VAN WiELINK P., 2011 - Calodromius bifasciatus and other Carabidae on 26 oak-trunks in a nature reserve in the Netherlands. ZooKeys, 100: 533-544.

FoCARILE A., 1973 - Sulla Coleotterofauna alticola del Gran San Bernardo (versante valdostano). Annali della Facoltà di Agraria dell'Universita di Torino, 9: 51-118.

FoCARILE A., 1974 - Aspetti zoogeografici del popolamento di Coleotteri (Insecta) nella Valle d'Aosta. Revue Valdôtaine d'Histoire Naturelle, 28: 5-53.

FoCARILE A., 1975 - Alcuni interessanti Coleotteri della Valle d'Aosta. Revue Valdôtaine d'Histoire Naturelle, 29 : 8-52.

FOCARILE A., 1976 - Sulla Coleotterofauna alticola della conca del Breuil (Valtournenche) e osservazioni sul popolamento pioniero delle zone di recente abbandono glaciale. Revue Valdôtaine d'Histoire Naturelle, 30: 126-168.

FoCARILE A., 1987 - Ecologie et Biogeographie des Coléoptères de haute altitude en Vallée d'Aoste. Regione Autonoma Valle d'Aosta. Assessorato Agricoltura, Foreste e Ambiente naturale, 167 pp., 72 carte, 4 tavv. 
Focarile A., Casale A., 1978 - Primi rilevamenti sulla Coleotterofauna alticola del Vallone di Clavalité (Fenis, Aosta). Revue Valdôtaine d'Histoire Naturelle, 32: 67-92.

GAud A., 1902 - Course du 28 juillet au 2 août 1902. Note entomologique. Bulletin de la Murithienne, 31:83-86.

HENRY J., 1915 - Quelques Coléoptères. Bulletin de la Société de la Flore Valdôtaine, 10: 65-78.

HeNRY J., 1916 - Petites contributions à l'histoire naturelle valdôtaine. Bulletin de la Société de la Flore Valdôtaine, 11: 35-46.

HenRY J., 1935 - Autre petite contribution à l'étude des Coléoptères de la Vallée d'Aoste. Bulletin de la Société de la Flore Valdôtaine, 22: 54-59.

HENRY J., 1938 - Autre contribution à l'étude des Coléoptères de la Vallée d'Aoste. Bulletin de la Société de la Flore Valdôtaine 23: $65-71$.

IEHL F., 1909 - Contribution à la Faune des Coléoptères valdotains. Bulletin de la Société de la Flore Valdôtaine, 5: 78-84.

Magistretti M., 1965 - Fauna d'Italia, VIII. Coleoptera: Cicindelidae, Carabidae. Catalogo topografico. Calderini, Bologna, 512 pp.

MAnZotti P., BALLĖvre M., 2014 - I travertini post-glaciali della bassa valle del Buthier, a nord di Aosta, con note sulle caratteristiche geologiche e morfologiche dell'area. Revue Valdotaine d'Histoire Naturelle, 68: 5-16.

Marggi W., Chittaro Y., Moretti M., 2010 - Harpalus fuscipalpis Sturm, 1818 und H. fuscicornis Ménétriés, 1832 (Coleoptera, Carabidae) in der Schweiz. Mitteilungen der Schweizerischen Entomolögischen Gesellschaft, 83: 11-15.

Mercalli L. (coord.), 2003 - Atlante climatico della Valle d'Aosta. Regione Autonoma Valle d'Aosta. Direzione Protezione civile, Ufficio Meteorologico, $405 \mathrm{pp}$.

Neri P., Bonavita P., Gudenzi I., Magrini P., Toledano L., 2011 - Bembidiina della fauna italo-corsa: chiavi di identificazione (Insecta Coleoptera Carabidae). Quaderno di Studi e Notizie di Storia Naturale della Romagna, 33: 1-183.

Neri P., Bonavita P., Vigna Taglianti A., Gudenzi I., 2013 - Note tassonomiche nomenclatoriali (3 contributo) su alcuni Bembidiina della Fauna Italiana (Insecta Coleoptera Carabidae). Quaderno di Studi e Notizie di Storia Naturale della Romagna, 36(2012): 89-95.

PAvesi P., 1904 - Esquisse d'une Faune Valdôtaine. Atti della Società Italiana di Scienze Naturali, Milano, 43: 199-260.

Ponel P., Coulon J., 2013 - Harpalus fuscicornis Ménétriès, 1832 et Harpalus fuscipalpis Sturm, 1818 tous deux présents en France (Coleoptera Carabidae). L'Entomologiste, 69(6): 323-325.

Ravizza C., 1972 - I Bembidion popolanti gli orizzonti montano, subalpino e alpino della Valle d'Aosta. Studi sui Bembidion. IX Contributo (Coleoptera Carabidae). Memorie della Società Entomologica Italiana, 51: 94-122.

Roda M., ZuCALI, M., 2008 - Meso and microstructural evolution of the Mont Morion metaintrusives complex (Dent Blanche nappe, Austroalpine domain, Valpelline, Western Italian Alps). Italian Journal of Geosciences, 127: 105-123.

SCheffler I., 1997 - Zur Verbreitung und Ökologie der Dromius-Arten im Potsdamer Stadtgebiet (Col., Carabidae). Entomologische Nachrichten und Berichte, 41: 179-182.

Schatzmayr A., 1930 - I Pterostichus italiani. Memorie della Società Entomologica Italiana, 8(1929): 145-339.

SCIAKY R., 1987 - Revisione delle specie paleartiche occidentali del genere Ophonus Dejean 1821 (Coleoptera Carabidae) (XVIII contributo alla conoscenza dei Coleoptera Carabidae). Memorie della Società Entomologica Italiana, 65(1986): 29-120.

Vigna Taglianti A., 2005 - Checklist e corotipi delle specie di Carabidae della fauna italiana. Appendice B, pp. 186-225. In: Brandmayr P., Zetto T., Pizzolotto R., (eds.), I Coleotteri Carabidi per la valutazione ambientale e la conservazione della biodiversità. Manuale operativo: APAT, Manuali e Linee Guida, 34: 240 pp. 